\title{
Geometric subgroups of mapping class groups
}

\author{
Luis Paris Dale Rolfsen
}

December 18, 2013

\begin{abstract}
This paper is a study of the subgroups of mapping class groups of Riemann surfaces, called "geometric" subgroups, corresponding to the inclusion of subsurfaces. Our analysis includes surfaces with boundary and with punctures. The centres of all the mapping class groups are calculated. We determine the kernel of inclusion-induced maps of the mapping class group of a subsurface, and give necessary and sufficient conditions for injectivity. In the injective case, we show that the commensurability class of a geometric subgroup completely determines up to isotopy the defining subsurface, and we characterize centralizers, normalizers, and commensurators of geometric subgroups.
\end{abstract}

Mathematics Subject Classification: Primary 57N05; Secondary 20F38

\section{Introduction}

Throughout the paper, $M$ will denote a compact, connected, oriented surface. The boundary $\partial M$, if nonempty, is a finite collection of simple closed curves. Consider a finite subset $P=\left\{p_{1}, \ldots, p_{m}\right\}$, of $m$ distinct points (often called "punctures" or "marked points") in the interior of $M$. Define $\mathcal{H}(M, P)$ to be the group of orientation-preserving homeomorphisms $h: M \rightarrow M$ such that $h$ is the identity on each boundary component of $M$ and $h(P)=P$. Our main object of study is the mapping class group $\mathcal{M}(M, P)=\pi_{0}(\mathcal{H}(M, P))$, the set of isotopy classes of these mappings, with composition as the group operation. We emphasize that throughout an isotopy, the boundary, and also the points $P$ remain fixed. It is clear that, up to isomorphism, these groups do not depend on the choice of $P$, but depend only on the cardinality $m=|P|$, so we may write $(M, m)$ or $\mathcal{M}(M, m)$ in place of $(M, P)$ or $\mathcal{M}(M, P)$, and simply $\mathcal{M}(M)$ for $\mathcal{M}(M, \emptyset)$. $\mathcal{M}(M, P)$ may 
equivalently be considered as the group of orientation-preserving diffeomorphisms of $(M, P)$, up to smooth isotopy. We refer the reader to the survey articles [2], [14], 15], 30] and [31] and their bibliographies for more information.

Let $N \subset M$ be a subsurface, by which we mean a closed subset which is also a surface and for which we always assume the further properties: (1) every component of $\partial N$ lies in the interior of $M,(2) P \cap \partial N=\emptyset$.

The inclusion $i:(N, N \cap P) \rightarrow(M, P)$ induces a natural mapping

$$
i_{*}: \mathcal{M}(N, N \cap P) \rightarrow \mathcal{M}(M, P) .
$$

If $[h]$ is a class of a homeomorphism of $N$, then $i_{*}([h])$ is represented by extending $h$ to $M$ using the identity mapping on $M \backslash N$. The image $i_{*}(\mathcal{M}(N, N \cap P))$ will be called a geometric subgroup of $\mathcal{M}(M, P)$.

Our study of these subgroups depends on a careful analysis of curves in $M$ and Dehn twists, which are the subject of Section 3. The mapping $i_{*}$ is often, but not always, injective. We determine its kernel in Section 4 . Section 5 is devoted to the centres of mapping class groups. These are certainly well-known to specialists and many of them can be found in the literature (see [15] and [17]). However, we need the general result for the remainder of the paper and the proofs are straightforward applications of Section 3. Our main result is that, assuming injectivity of the $i_{*}$, up to a finite number of exceptions, two geometric subgroups are commensurable if and only if they are equal if and only if their respective defining subsurfaces are isotopic (Theorem 6.5). Note that the assumption that $\partial N$ lies entirely in the interior of $M$ is necessary for the conclusion of Theorem 6.5; indeed, without this assumption, it is very easy to construct non-isotopic subsurfaces $N$ and $N^{\prime}$ which define the same geometric subgroup, $N$ satisfying $\partial N \cap \partial M=\emptyset$ and $N^{\prime}$ satisfying $\partial N^{\prime} \cap \partial M \neq \emptyset$. From the main result, still assuming injectivity of the $i_{*}$, we characterize the commensurator, the normalizer and the centralizer of a geometric subgroup in $\mathcal{M}(M, P)$.

We close this introduction and illustrate the injectivity question by discussing some basic examples, which are well-known (c.f. [1] and [7]).

Examples: $(1) \mathcal{M}\left(D^{2}\right) \cong\{1\}$ and $\mathcal{M}\left(D^{2}, 1\right) \cong\{1\}$, where $D^{2}$ is a disk.

(2) Similarly, for the 2-sphere, $\mathcal{M}\left(S^{2}\right)$ and $\mathcal{M}\left(S^{2}, 1\right)$ are trivial.

The above examples are essentially the only surface mapping class groups which are trivial.

(3) $\mathcal{M}\left(S^{1} \times I\right) \cong \mathbf{Z}$.

The mapping class group of the annulus $S^{1} \times I$ is generated by a Dehn twist, described in Section 3. 
(4) $\mathcal{M}\left(S^{1} \times I, 1\right) \cong \mathbf{Z}^{2}$. Here, Dehn twists along the two boundary components are generators. They are not isotopic because of the puncture.

(5) As a family of examples, consider disks $D_{1} \subset D_{2} \subset \ldots$ in the complex plane, where the diameter of $D_{m}$ is taken to be the real line interval $[1-m /(m+1), m+1 / 2]$. Take $P_{m}=D_{m} \cap \mathbf{Z}=\{1, \ldots, m\}$. Then it is also well-known that the mapping class groups $\mathcal{M}\left(D_{m}, P_{m}\right)$ are isomorphic with the classical braid groups $B_{m}$ of Artin, which have generators $\sigma_{1}, \ldots, \sigma_{m-1}$ subject to the relations

$$
\sigma_{i} \sigma_{j}=\sigma_{j} \sigma_{i}, \quad|i-j|>1, \quad \sigma_{i} \sigma_{i+1} \sigma_{i}=\sigma_{i+1} \sigma_{i} \sigma_{i+1} .
$$

Under the isomorphism, $\sigma_{j}, \quad 0<j<m$ corresponds with the (class of the) diffeomorphism consisting of a "half-twist" interchanging the integers $j$ and $j+1$, and supported on a small neighborhood of the interval $[j, j+1] \subset$ C. See [i] and [7] for details, but beware some differences in choice of conventions. It is classical, but nontrivial, that for $n<m$ the homomorphism $B_{n} \rightarrow B_{m}$, taking $\sigma_{j} \in B_{n}$ to $\sigma_{j} \in B_{m}$ is injective, allowing us to write $B_{n} \subset B_{m}$. We chose notation so that under the isomorphisms, $B_{n} \rightarrow B_{m}$ corresponds to the inclusion-induced mapping $i_{*}: \mathcal{M}\left(D_{n}, P_{n}\right) \rightarrow \mathcal{M}\left(D_{m}, P_{m}\right)$. We conclude that in this case $i_{*}$ is injective. Note that the closure of the complementary subsurface is an annulus with $m-n$ punctures. The commensurator, the normalizer and the centralizer of $\mathcal{M}\left(D_{n}, P_{n}\right)$ in $\mathcal{M}\left(D_{m}, P_{m}\right)$ are characterized in [8] and [35].

(6) The following is an example of an inclusion map which is not injective on mapping class groups. Take $M=S^{2}$ a 2-sphere with $P=2$ points in $S^{2}$, and let $D$ be a disk in $S^{2}$ which encloses the points $P$. We have the map $i_{*}: \mathcal{M}(D, P) \rightarrow$ $\mathcal{M}\left(S^{2}, P\right)$. As already discussed, $\mathcal{M}(D, P)$ is the braid group $B_{2}$, which is infinite cyclic, generated by $\sigma_{1}$. However, $\sigma_{1}^{2}$ is isotopic with a Dehn twist along $\partial D$. In the larger surface $M=S^{2}$ this twist is isotopic with the identity (rel $P$ ). So the kernel of $i_{*}$ in this case is the infinite cyclic subgroup of index 2 in $\mathcal{M}(D, P) . \mathcal{M}\left(S^{2}, 2\right)$ is cyclic of order 2 .

(7) For the torus $T^{2}=S^{1} \times S^{1}$ with either zero or one puncture, the mapping class group is the modular group of invertible $2 \times 2$ matrices with integer entries: $\mathcal{M}\left(T^{2}\right) \cong \mathcal{M}\left(T^{2}, 1\right) \cong S L(2, \mathbf{Z})$. Dehn twists along the curves $S^{1} \times *$ and $* \times S^{1}$ generate the mapping class group. Note that if $A$ is an annulus neighborhood of one of these curves, and happens to enclose the puncture of $\mathcal{M}\left(T^{2}, 1\right)$, the map $i_{*}: \mathcal{M}(A, 1) \rightarrow \mathcal{M}\left(T^{2}, 1\right)$ fails to be injective. 


\section{Subgroups of mapping class groups}

In this section we review some of the literature regarding subgroups of mapping class groups. Although there is little published on the geometric subgroups, which are the main concern of the present paper, certain other subgroups are quite well understood. First, we recall some general properties of the mapping class groups themselves. A more complete survey can be found in [30].

For the closed surface $M_{g}$ of genus $g$, the mapping class groups $\mathcal{M}\left(M_{g}, m\right)$ are known to be finitely presented [9], [11], [23] [25], [26], [29], [36], [37]. The generators can be taken to be Dehn twists (discussed below) along curves and half-twists along arcs connecting the punctures.

According to Grossman [10] and Ivanov [15], $\mathcal{M}\left(M_{g}, m\right)$ is residually finite for every nontrivial element, there is a homomorphism of the mapping class group onto a finite group which does not kill that element. This implies that $\mathcal{M}\left(M_{g}, m\right)$ is Hopfian ([24], Chapter IV) - every epimorphism $\mathcal{M}\left(M_{g}, m\right) \rightarrow \mathcal{M}\left(M_{g}, m\right)$ is an isomorphism. Conversely, in a recent paper [17] Ivanov and McCarthy proved that $\mathcal{M}\left(M_{g}, m\right)$ is co-Hopfian - every monomorphism $\mathcal{M}\left(M_{g}, m\right) \rightarrow \mathcal{M}\left(M_{g}, m\right)$ is an isomorphism.

Although, it contains torsion elements, $\mathcal{M}\left(M_{g}\right)$ has a finite index subgroup which is torsion free (see [30] for a sketch of a proof.) It has recently been shown by Mosher [32] that the mapping class groups are automatic. This implies that the word problem is solvable (in quadratic time) and many other consequences [6].

The outer automorphism group of $\mathcal{M}\left(M_{g}, m\right)$ has been determined by Ivanov [16] and McCarthy [28]. It is equal to $\mathbf{Z} / 2 \mathbf{Z}$ under the assumptions $g \neq 0, m \geq 3$ if $g=1$, and $m \geq 1$ if $g=2$.

The abelianization of $\mathcal{M}\left(M_{g}\right)$ is cyclic of order 12 , when $g=1$, cyclic of order 10 when $g=2$, and trivial (that is, $\mathcal{M}\left(M_{g}\right)$ is perfect) for $g>2$ 34].

Finite subgroups of $\mathcal{M}\left(M_{g}\right)$ have been extensively studied. The so-called Nielsen realization problem [38] was solved by Kerckhoff [22]. It asserts that for any finite subgroup $F$ of $\mathcal{M}\left(M_{g}\right)$, there is a complex structure on $M_{g}$ such that $F$ is realized as a group of holomorphic automorphisms of $M_{g}$. According to a classical result of Hurwitz [12], the orders of finite subgroups are bounded: $|F| \leq 84(g-1), g>1$.

McCarthy [27] showed that subgroups of $\mathcal{M}\left(M_{g}\right)$ satisfy the Tits alternative: every subgroup either contains a free group on two generators, or a solvable subgroup of finite index. Birman, Lubotzky and McCarthy [3] proved that solvable subgroups of $\mathcal{M}\left(M_{g}\right)$ are virtually abelian, and gave upper bounds for the rank of free abelian subgroups. Ivanov [15] proved these two results for $\mathcal{M}\left(M_{g}, m\right)$ and showed that: if $G$ is a subgroup of $\mathcal{M}\left(M_{g}, m\right)$ which is not virtually abelian, then 
$G$ contains an uncountably infinite number of maximal subgroups of infinite index. The Frattini subgroup $\phi(G)$ of a group $G$ is the intersection of all its maximal subgroups. Ivanov also proved that the Frattini subgroup of a finitely generated subgroup of $\mathcal{M}\left(M_{g}, m\right)$ is nilpotent. Note that this property also holds for finitely generated linear groups [33].

The centres of the $\mathcal{M}\left(M_{g}, m\right)$ are well-known [15], [17]: cyclic of order two if $g=1$ and $m \leq 2$, and if $g=2$ and $m=0$, and trivial otherwise. We determine the centres in the more general situation, with boundary, in the present paper.

The Torelli subgroup of the mapping class group consists of classes of mappings which induce the identity on the homology of the surface. These subgroups have been studied extensively in the series of papers [18], [19], [20], [21]. In particular, they describe a finite set of generators for the Torelli groups. The cohomological properties of mapping class groups and the Torelli groups have been studied intensively and are also well-described in [30] and [31]. We shall not use any of these deeper properties - indeed our methods are quite elementary and self-contained, requiring as background only some basic properties of curves on surfaces due to Epstein [5].

\section{Curves and Dehn twists}

Working within the context of a surface $M$ with punctures $P$ as described above, we shall consider a simple closed curve in $M \backslash P$ as an embedding $c: S^{1} \rightarrow M \backslash P$ which does not intersect the boundary of $M$. Note that $c$ has an orientation; the curve with the opposite orientation, but same image will be denoted $c^{-1}$. By abuse of notation, we also use the symbol $c$ to denote the image of $c$. We will say that $c$ is essential if it does not bound a disk in $M$ disjoint from $P$, and that $c$ is generic if it does not bound a disk in $M$ containing 0 or 1 point of $P$.

Two simple closed curves $a, b$ are isotopic if there exists a continuous family $h_{t} \in \mathcal{H}(M, P), \quad t \in[0,1]$ of homeomorphisms such that $h_{0}$ is the identity and $h_{1} \circ a=b$. Isotopy of curves is an equivalence relation which we denote by $a \simeq b$. Following [7] the index of intersection of two simple closed curves $a$ and $b$ is:

$$
I(a, b)=\inf \left\{\left|a^{\prime} \cap b^{\prime}\right| ; a^{\prime} \simeq a, b^{\prime} \simeq b\right\}
$$

We note that:

1) $I(a, b)=\inf \left\{\left|a^{\prime} \cap b\right| ; a^{\prime} \simeq a\right\}$;

2) If $a$ is not generic, then $I(a, b)=0$ for every simple closed curve $b$;

3) If $a \simeq b$ then $I(a, b)=0$. 
A bigon cobounded by two simple closed curves $a$ and $b$ in $M \backslash P$ is a disk $D \subset M \backslash P$ whose boundary is the union of an arc of $a$ and an arc of $b$.

Proposition 3.1 (Epstein [5]) Let $a, b: S^{1} \rightarrow M \backslash P$ be two essential simple closed curves, and suppose $a$ is isotopic to $b$.

i) If $a \cap b=\emptyset$, then there exists an annulus in $M \backslash P$ whose boundary components are $a$ and $b$.

ii) If $a \cap b \neq \emptyset$, and they intersect transversely, then $a$ and $b$ cobound a bigon.

Proposition 3.2 Let $a, b: S^{1} \rightarrow M \backslash P$ be two essential simple closed curves, which intersect transversely. Then

$$
I(a, b)=|a \cap b|
$$

if and only if $a$ and $b$ do not cobound a bigon.

Proof: It is clear that if $a$ and $b$ cobound a bigon, one can isotop one of the curves across the bigon and reduce the cardinality of the intersection by two. Now suppose they do not cobound a bigon, and choose a simple closed curve $a^{\prime}$ isotopic to $a$ and transverse to $a$ such that

$$
\left|a^{\prime} \cap b\right|=I(a, b) .
$$

We will argue by induction on $\left|a^{\prime} \cap a\right|$ that

$$
|a \cap b|=\left|a^{\prime} \cap b\right|=I(a, b) .
$$

If $\left|a^{\prime} \cap a\right|=0$, then by Proposition 3.1, there is an annulus in $M \backslash P$ with boundary components $a$ and $a^{\prime}$. Each arc of intersection of $b$ with the annulus must run from one boundary component to the other (see Figure 1), since neither $a, b$ nor $a^{\prime}, b$ cobound bigons. Therefore $|a \cap b|=\left|a^{\prime} \cap b\right|=I(a, b)$.

Now suppose $\left|a^{\prime} \cap a\right|>0$. By Proposition 3.1, $a$ and $a^{\prime}$ cobound a bigon. We may assume $a^{\prime} \cap a \cap b$ is empty, so any arc of intersection of $b$ with the bigon must have one endpoint in $a$ and the other in $a^{\prime}$ (see Figure 1), again because neither $a, b$ nor $a^{\prime}, b$ cobound bigons. Therefore we may push $a^{\prime}$ across the bigon to obtain a new curve $a^{\prime \prime}$ isotopic to $a$ and satisfying:

$$
\left|a^{\prime \prime} \cap a\right|=\left|a^{\prime} \cap a\right|-2 \quad \text { and } \quad\left|a^{\prime \prime} \cap b\right|=\left|a^{\prime} \cap b\right|=I(a, b) .
$$

By inductive hypothesis $|a \cap b|=\left|a^{\prime \prime} \cap b\right|=I(a, b)$. 


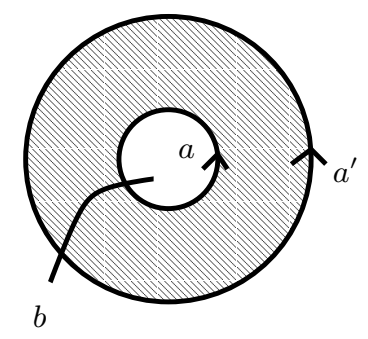

$a \cap a^{\prime}=\emptyset$

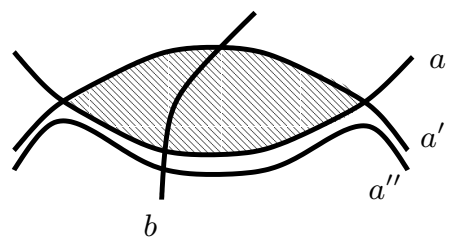

$a \cap a^{\prime} \neq \emptyset$

Figure 1: Curves cobounding an annulus and a bigon.

Definition: If we parametrize $S^{1}$ as the unit circle in the complex plane, and the interval $I=[0,1]$, then the prototype Dehn twist $\tau: S^{1} \times I \rightarrow S^{1} \times I$ is given by

$$
\tau(z, t):=\left(z e^{2 \pi i t}, t\right)
$$

Note that $\tau$ is the identity on the boundary circles. More generally, let $a$ : $S^{1} \rightarrow M \backslash P$ be a simple closed curve, and let $N \subset M \backslash P$ be an annulus regular neighborhood of the image of $a$, parametrized by $\tilde{a}: S^{1} \times I \rightarrow N$. Define the Dehn twist along $a$ to be (the isotopy class of) the homeomorphism $A(x)=\tilde{a} \tau \tilde{a}^{-1}(x)$ for $x \in N, A(x)=x$ for $x$ outside $N$.

We will use the convention throughout that a curve is denoted by a lower case letter and a Dehn twist along the curve is denoted by the corresponding capital letter. Note that, depending on parametrization chosen, there are two choices of Dehn twist along $a$, inverse to each other. Usually, the choice is immaterial, provided one is consistent throughout, but we will adopt the convention in illustrations that a curve crossing $a$ will make a "right turn" at each encounter with $a$, after being acted on by $A$ (see Figure 2). We also observe:

1) The Dehn twist along $a^{-1}$ coincides with the Dehn twist along $a$.

2) The curve $a$ is fixed by the Dehn twist $A$.

3 ) If two curves are isotopic, then so are their corresponding Dehn twists.

4) If $h$ is a homeomorphism of $M$, the Dehn twist along $h(a)$ is $h A h^{-1}$.

5) If $a$ is not generic, $A$ is isotopic to the identity.

Proposition 3.3 Let $a, b: S^{1} \rightarrow M \backslash P$ be two simple closed curves, $A$ the Dehn twist along $a$ and $n$ any integer. Then

$$
I\left(A^{n}(b), b\right)=|n| \cdot I(a, b)^{2} .
$$




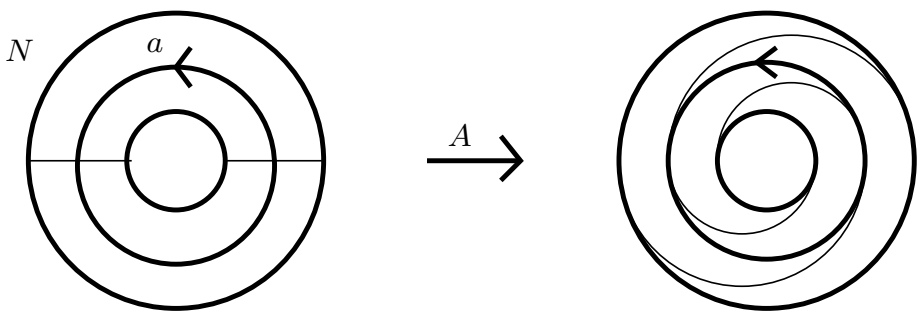

Figure 2: Dehn twist along curve $a$.

Proof: This is a special case of a formula in [7]. We outline a proof, leaving details to the reader. Assume $|a \cap b|=I(a, b)$. The cases $n=0$ or $I(a, b)=0$ being trivial, suppose they are nonzero. Construct the curve $A^{n}(b)$, which can be seen to cross $b$ exactly $|n| I(a, b)$ times at each point of intersection of $a$ with $b$ (see Figure 3). The proof is completed by noting that this is the minimal intersection of $A^{n}(b)$ with $b$, up to isotopy. For otherwise, by Proposition 3.2 there would be a bigon cobounded by them. One can see this would imply that $a$ and $b$ also cobound a bigon, which is impossible, again by Proposition 3.2 .

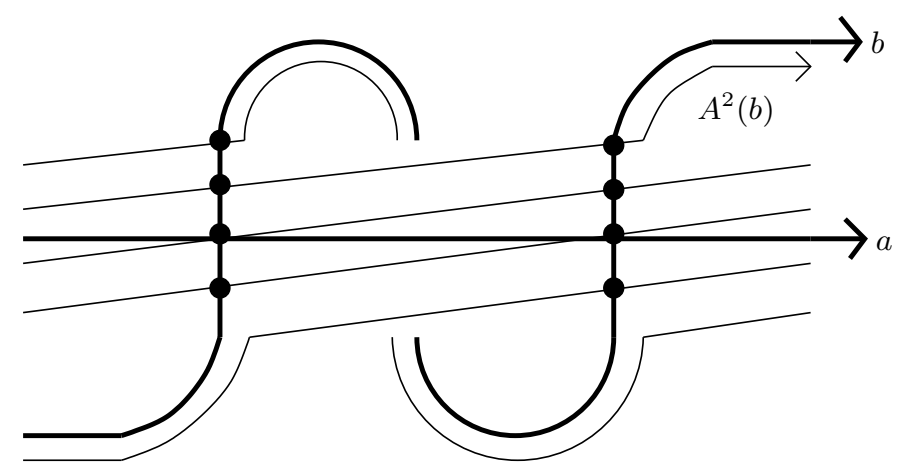

Figure 3: Intersection of $b$ with $A^{2}(b)$.

Proposition 3.4 Suppose $a_{1}, \ldots, a_{p}: S^{1} \rightarrow M \backslash P$ are generic simple closed curves such that:

a) $a_{i} \cap a_{j}=\emptyset$ if $i \neq j$,

b) $a_{i}$ is neither isotopic with $a_{j}$ nor $a_{j}^{-1}$, if $i \neq j$,

c) none of the $a_{i}$ is isotopic with a boundary component of $M$.

Then for each $i, 1 \leq i \leq p$, there exists a simple closed curve $b: S^{1} \rightarrow M \backslash P$ such that $a_{j} \cap b=\emptyset$ if $i \neq j$, and $\left|a_{i} \cap b\right|=I\left(a_{i}, b\right)>0$. 
Remark: The last condition implies that $b$ must be generic in $M \backslash P$.

Proof: We "cut open" $M$ along all the curves $a_{i}$ to obtain the connected compact surfaces $N_{1}, \ldots, N_{r}$ with the property that the union of the interiors of the $N_{j}$ is the interior of $M \backslash \bigcup_{i=1}^{p} a_{i}$ and each boundary component of $N_{j}$ is either a boundary component of $M$ or a copy of some $a_{i}$. There is a continuous projection of the disjoint union onto $M$ :

$$
\rho: \coprod_{j=1}^{r} N_{j} \rightarrow M
$$

which covers each curve $a_{i}$ twice, and is injective on the union of the interiors of the $N_{j}$. Now fix $i \in\{1, \ldots, r\}$ and consider the curve $a_{i}=\rho\left(c_{1}\right)=\rho\left(c_{2}\right)$, where $c_{1}$ is a component of the boundary of some $N_{j}$ and $c_{2}$ is a component of the boundary of $N_{k}$.

Case $1, j=k$ : Then $c_{1}$ and $c_{2}$ are different components of the boundary of the connected surface $N_{j}$. There is an $\operatorname{arc} \tilde{b}$ in $N_{j}$ with one endpoint in $c_{1}$, the other endpoint being the point of $c_{2}$ having same projection in $a_{i}$, and the interior of $\tilde{b}$

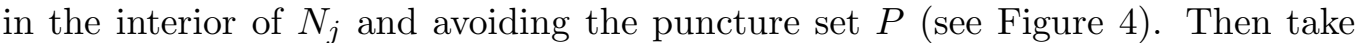
$b=\rho(\tilde{b})$. Clearly $a_{j} \cap b=\emptyset$ if $i \neq j$; moreover $a_{i}$ and $b$ are transverse and they cannot cobound a bigon, so $\left|a_{i} \cap b\right|=1=I\left(a_{i}, b\right)$.

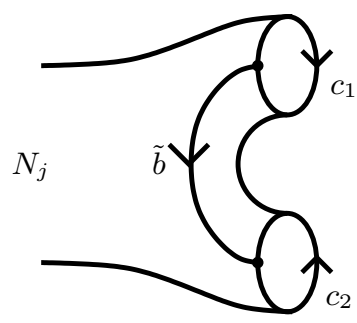

Figure 4: Constructing $b$, case 1 .

Case $2, j \neq k$ : Then $c_{1}$ is a boundary component of $N_{j}$, and by hypothesis, if $N_{j}$ is a disk, then $N_{j} \cap P$ contains at least two points, and if $N_{j}$ is an annulus, $N_{j} \cap P$ contains at least one point. So in any case, there exists an $\operatorname{arc} b_{1}$ in $N_{j}$ with both endpoints in $c_{1}$, interior in the interior of $N_{j}$ and disjoint from $P$, and such that $b_{1}$ and $c_{1}$ do not cobound a bigon in $N_{j} \backslash P \cap N_{j}$ (see Figure 國). In the same way we choose an arc $b_{2}$ in $N_{k}$, whose endpoints in $c_{2}$ project to $\rho\left(b_{1} \cap c_{1}\right)$ in $a_{i}$, whose interior is interior to $N_{k} \backslash P \cap N_{k}$, and such that $b_{2}$ and $c_{2}$ do not cobound a bigon in $N_{k} \backslash P \cap N_{k}$. Define $b=\rho\left(b_{1} \cup b_{2}\right)$. Then $a_{j} \cap b=\emptyset$ if $i \neq j$ 


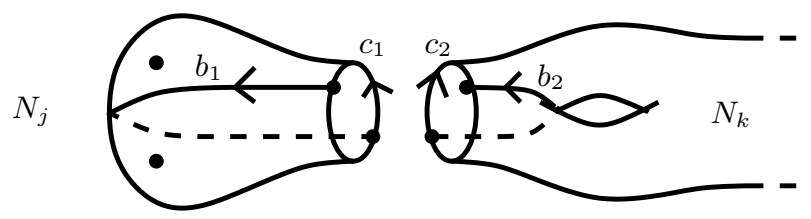

Figure 5: Constructing $b$, case 2 .

and $\left|a_{i} \cap b\right|=I\left(a_{i}, b\right)=2$, because we have arranged that $a_{i}$ and $b$ do not cobound a bigon in $M \backslash P$.

Now consider a subsurface $N \subset M$; recall this includes the assumption that $P \cap \partial N=\emptyset$ and $\partial N$ is interior to $M$. We will say that $N$ is essential if each component of $\overline{M \backslash N}$ which is a disk has nonempty intersection with the puncture set $\mathrm{P}$.

A component $N^{\prime}$ of $\overline{M \backslash N}$ will be called an exterior cylinder if $N^{\prime}$ is a cylinder (= annulus) disjoint from $P$, with both components of $\partial N^{\prime}$ also being components of $\partial N$ (see Figure 6).

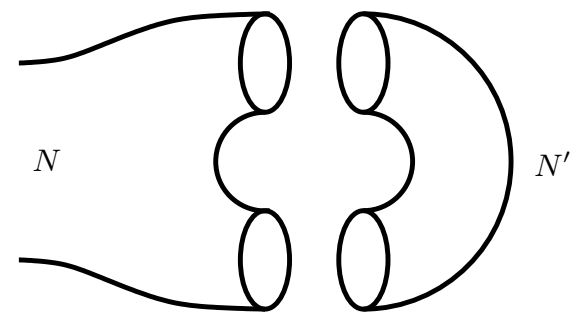

Figure 6: $N^{\prime}$ is an exterior cylinder for $N$.

Proposition 3.5 Let $N \subset M$ be an essential subsurface and let $a, b: S^{1} \rightarrow$ $N \backslash N \cap P$ be essential simple closed curves. Assume that $a$ is not isotopic in $N \backslash N \cap P$ to a boundary component of an exterior cylinder. Then $a$ and $b$ are isotopic in $M \backslash P$ if and only if they are isotopic in $N \backslash N \cap P$.

Proof: The only nontrivial part is to show that if $a$ and $b$ are isotopic in $M \backslash P$, then they are isotopic in $N \backslash N \cap P$. We assume $a$ and $b$ intersect transversely and argue by induction on $|a \cap b|$.

If $|a \cap b|=0$, then Proposition 3.1 implies there exists an annulus in $M \backslash P$ with (unoriented) boundary $a \cup b$. Since $a$ is not isotopic to a boundary component 
of an exterior cylinder and $N$ is essential, the annulus is disjoint from $\partial N$, and therefore it lies in $N \backslash N \cap P$. It follows that $a$ and $b$ are isotopic in $N \backslash N \cap P$.

If $|a \cap b|>0$, then $a$ and $b$ cobound a bigon in $M \backslash P$, by Proposition 3.1. Because $N$ is essential, the bigon is disjoint from $\partial N$, and therefore it lies in $N$. Pushing across this bigon defines an isotopy in $N \backslash P$ from $b$ to a curve $b^{\prime}$ with $\left|b^{\prime} \cap a\right|=|b \cap a|-2$. By inductive hypothesis, $b^{\prime}$ is isotopic with $a$ in $N \backslash N \cap P$, so the same is true of $b$.

Proposition 3.6 Consider two generic simple closed curves $a, b: S^{1} \rightarrow M \backslash P$, and let $A$ and $B$, respectively, denote Dehn twists along these curves. If $j$ and $k$ are integers, $j \neq 0$, such that $A^{j}=B^{k}$ in $\mathcal{M}(M, P)$, then a is isotopic to $b$ or $b^{-1}$ in $M \backslash P$.

Remark: This proposition and the next one can be found in [17] for $\partial M=\emptyset$. The general case needs an extra argument to consider Dehn twists along boundary curves.

Proof: We will argue that if $a$ is not isotopic with $b^{ \pm 1}$ then $A^{j} \neq B^{k}$. It may be assumed that $a$ and $b$ meet transversely and that $|a \cap b|=I(a, b)$. First assume $I(a, b)>0$. Then, by Proposition 3.3,

$$
\begin{gathered}
I\left(A^{j}(b), b\right)=|j| I(a, b)^{2}>0 \\
I\left(B^{k}(b), b\right)=I(b, b)=0
\end{gathered}
$$

and we conclude $A^{j} \neq B^{k}$.

Now suppose that $I(a, b)=0$. If $M$ has nonempty boundary, consider the larger closed surface $\hat{M}$ obtained by gluing a torus minus a disk to each boundary component of $M$ (if $\partial M=\emptyset$, let $\hat{M}=M$ ). By Proposition 3.5 $a$ is not isotopic with $b^{ \pm 1}$ in $\hat{M} \backslash P$. By Proposition 3.4 there is a simple closed curve $c$ in $\hat{M} \backslash P$ such that $b \cap c=\emptyset$ and $|a \cap c|=I(a, c)>0$. Then

$$
\begin{gathered}
I\left(A^{j}(c), c\right)=|j| I(a, c)^{2}>0 \\
I\left(B^{k}(c), c\right)=I(c, c)=0,
\end{gathered}
$$

and therefore $A^{j} \neq B^{k}$ in $\mathcal{M}(\hat{M}, P)$; so $A^{j} \neq B^{k}$ in $\mathcal{M}(M, P)$.

Proposition 3.7 Consider two generic simple closed curves $a, b: S^{1} \rightarrow M \backslash P$, and let $A$ and $B$, respectively, denote Dehn twists along these curves. If $j$ and $k$ are integers, $j \neq 0$ and $k \neq 0$, such that $A^{j}$ and $B^{k}$ commute in $\mathcal{M}(M, P)$, then $I(a, b)=0$. 
Proof: Assuming $A^{j}$ and $B^{k}$ commute, we have

$$
A^{j}=B^{k} A^{j} B^{-k}=C^{j},
$$

where $C$ is the Dehn twist along the curve $c=B^{k}(a)$. By Proposition 3.6 it follows that $c$ is isotopic with $a^{ \pm 1}$. Proposition 3.3 implies

$$
0=I\left(a^{ \pm 1}, a\right)=I(c, a)=I\left(B^{k}(a), a\right)=|k| I(a, b)^{2},
$$

so $I(a, b)=0$.

Remark: An alternative proof of Proposition 3.7 can be deduced from [13], where it is shown that $A$ and $B$ commute if $I(a, b)=0, A$ and $B$ satisfy the braid relation $A B A=B A B$ if $I(a, b)=1$, and $A$ and $B$ generate a free group if $I(a, b) \geq 2$.

Proposition 3.8 Suppose $a_{1}, \ldots, a_{p}: S^{1} \rightarrow M \backslash P$ are generic simple closed curves which are pairwise disjoint, and no curve $a_{i}$ is isotopic to $a_{j}$ or $a_{j}^{-1}, i \neq j$. Consider the function

$$
h: \mathbf{Z}^{p} \rightarrow \mathcal{M}(M, P)
$$

defined by

$$
h\left(n_{1}, \ldots, n_{p}\right)=A_{1}^{n_{1}} \cdots A_{p}^{n_{p}},
$$

where $A_{i}$ is the Dehn twist about $a_{i}$. Then $h$ is an injective homomorphism.

Proof: Because the curves are disjoint, the Dehn twists commute, and $h$ is a homomorphism. To see it is injective, suppose $A_{1}^{n_{1}} \cdots A_{p}^{n_{p}}$ is the identity of $\mathcal{M}(M, P)$ for some $\left(n_{1}, \ldots, n_{p}\right)$. We again employ the trick of considering the closed surface $\hat{M}$, which is $M$ plus a copy of a torus minus a disk glued to each boundary component. Clearly, each $a_{i}: S^{1} \rightarrow \hat{M}$ is generic, and by Proposition $3.5 a_{i}$ is not isotopic to $a_{j}^{ \pm 1}$ in $\hat{M} \backslash P$, when $i \neq j$. Now fix an index $i \in\{1, \ldots, p\}$. Proposition 3.4 supplies a simple closed curve $b$ in $\hat{M} \backslash P$ disjoint from $a_{j}, \quad i \neq j$, with

$$
\left|a_{i} \cap b\right|=I\left(a_{i}, b\right)>0 .
$$

We calculate, using commutativity of the twists, $b \cap a_{j}=\emptyset, \quad i \neq j$, and Proposition 3.3 .

$$
\begin{aligned}
0 & =I(b, b)=I\left(A_{1}^{n_{1}} \cdots A_{p}^{n_{p}}(b), b\right) \\
& =I\left(A_{i}^{n_{i}}(b), b\right)=\left|n_{i}\right| I\left(a_{i}, b\right)^{2} .
\end{aligned}
$$

Therefore $n_{i}=0$ and we have shown $i_{*} \circ h$ is injective, where $i:(M, P) \rightarrow(\hat{M}, P)$, so $h$ is injective. 
Corollary 3.9 If $a: S^{1} \rightarrow M \backslash P$ is a generic simple closed curve, then the Dehn twist about a has infinite order in $\mathcal{M}(M, P)$.

Proposition 3.10 Let $a_{1}, \ldots, a_{p}, b_{1}, \ldots, b_{p}: S^{1} \rightarrow M \backslash P$ be essential simple closed curves satisfying:

1) $a_{i} \cap a_{j}=\emptyset$ and $b_{i} \cap b_{j}=\emptyset$ if $i \neq j$;

2) $a_{i}$ is not isotopic with $a_{j}^{ \pm 1}$ and $b_{i}$ is not isotopic with $b_{j}^{ \pm 1}$ if $i \neq j$;

3) $a_{i}$ is isotopic to $b_{i}$ for each $i=1, \ldots, p$.

Then there exists an isotopy $h_{t} \in \mathcal{H}(M, P)$ such that $h_{0}=i d$ and $h_{1} \circ a_{i}=b_{i}$ for all $i=1, \ldots, p$.

Proof: We will use a double induction. First, induction on $p$. The proposition is obvious if $p=1$, so we assume it is true for $p-1$ pairs of curves. This means that, replacing each $a_{i}$ by $h_{1} \circ a_{i}$, we may assume that $a_{i}=b_{i}$ for $i=1, \ldots, p-1$. Then we have $a_{p}$ disjoint from $a_{j}=b_{j}, \quad j<p$ and also $b_{p}$ disjoint from $a_{j}=b_{j}, \quad j<p$, and $a_{p}$ isotopic in $M \backslash P$ to $b_{p}$. We will be done if we show that there is a further isotopy taking $a_{p}$ to $b_{p}$ which does not move the curves $a_{j}=b_{j}, \quad j<p$. Taking $a_{p}$ and $b_{p}$ to be transverse, we will argue by induction on $\left|a_{p} \cap b_{p}\right|$.

If $\left|a_{p} \cap b_{p}\right|=0$, then $a_{p}$ and $b_{p}$ cobound an annulus in $M \backslash P$, by Proposition 3.1 . Any simple closed curve in this annulus must be either inessential or parallel to a boundary component, so our hypotheses guarantee that the annulus is disjoint from all the curves $a_{j}=b_{j}, \quad j<p$. Then there is an isotopy across the annulus taking $a_{p}$ to $b_{p}$; the isotopy may be taken to be the identity outside a small neighborhood of the annulus, so the other curves do not move. Suppose $\left|a_{p} \cap b_{p}\right|>0$, then by Proposition 3.1, the curves cobound a bigon in $M \backslash P$, and we may argue as above that the bigon is disjoint from the other curves. An isotopy taking $a_{p}$ across the bigon, fixed outside a neighborhood of the bigon, reduces the number $\left|a_{p} \cap b_{p}\right|$ and does not move the other curves. The inductive hypothesis now gives a final isotopy taking $a_{p}$ to $b_{p}$.

\section{Subsurfaces and injectivity}

Define the pure mapping class group of $M$ relative to $P$ to be the subgroup $\mathcal{P} \mathcal{M}(M, P)$ of $\mathcal{M}(M, P)$ consisting of all (classes of) diffeomorphisms which fix $P$ pointwise. Letting $\Sigma_{P}$ denote the group of permutations of the set $P$, we have the exact sequence

$$
1 \rightarrow \mathcal{P} \mathcal{M}(M, P) \rightarrow \mathcal{M}(M, P) \rightarrow \Sigma_{P} \rightarrow 1
$$


Definitions: A pair equivalent to $\left(D^{2}, 2\right)$ is called a pantalon of type $I$ (see Figure 7). We have already observed that $\mathcal{M}\left(D^{2}, 2\right)$ is infinite cyclic, generated by a "half-twist" $\sigma$ which interchanges the two punctures. $\mathcal{P} \mathcal{M}\left(D^{2}, 2\right)$ is the subgroup generated by $\sigma^{2}$, which is (up to isotopy) the same as a Dehn twist along the boundary.

A pair $\left(S^{1} \times I, 1\right)$ is a pantalon of type $I I$ (see Figure 1). The mapping class groups $\mathcal{M}\left(S^{1} \times I, 1\right)$ and $\mathcal{P} \mathcal{M}\left(S^{1} \times I, 1\right)$ coincide, and are isomorphic with $\mathbf{Z}^{2}$, generated by the Dehn twists along the two boundary components.

A pantalon of type $I I I$ is a connected planar surface $M$ with three boundary components, the puncture set $P$ is taken to be empty (see Figure 7 ). Its mapping class group is $\mathcal{M}(M, 0) \cong \mathbf{Z}^{3}$, generated by Dehn twists along the three boundary curves.

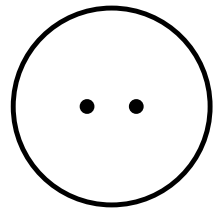

Type I

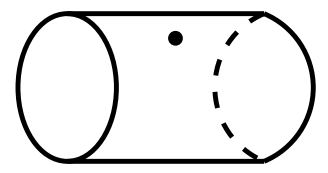

Type II

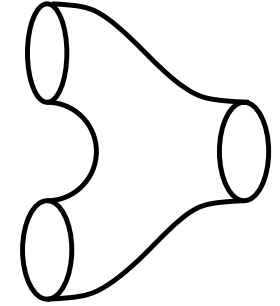

Type III

Figure 7: The three types of pantalons.

We now define one of our basic tools, a pantalon decomposition of a pointed surface $(M, P)$ (see Figure 8). This consists of:

1) a collection $\left(M_{i}, P_{i}\right), i=1, \cdots, r$, each pair being a pantalon of one of the three types, together with maps $\phi_{i}:\left(M_{i}, P_{i}\right) \rightarrow(M, P)$,

2) a collection $a_{1}, \cdots, a_{p}$ of simple closed curves in $M$, disjoint from each other, from $P$ and from $\partial M$ satisfying the following.

a) Each $\phi_{i}$ is injective on the interior of $M_{i}$.

b) $\phi_{i}\left(\right.$ int $\left.M_{i}\right)$ and $\phi_{j}\left(\right.$ int $\left.M_{j}\right)$ are disjoint if $i \neq j$.

c) $\phi_{i}$ takes each boundary component of $M_{i}$ to one of the curves $a_{k}$ or to a boundary component of $M$. Two boundary components of $M_{i}$ are allowed to map to the same curve $a_{k}$.

d) $M=\cup_{i=1}^{r} \phi_{i}\left(M_{i}\right)$ and $P=\cup_{i=1}^{r} \phi_{i}\left(P_{i}\right)$.

Informally, we say that $a_{1}, \cdots, a_{p}$ determine a pantalon decomposition of $(M, P)$, cutting $M$ open into pantalons $\left(M_{i}, P_{i}\right)$. 


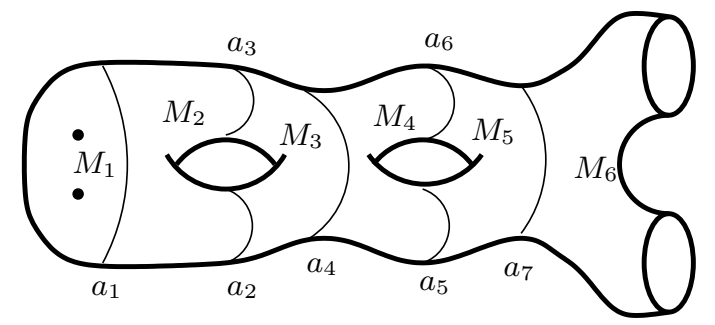

Figure 8: A pantalon decomposition.

It is straightforward to check that every connected compact orientable surface $M$ with punctures $P$ admits a pantalon decomposition, with the following exceptions:

a) $M=S^{2}$, a sphere, and $|P| \leq 3$;

b) $M=D^{2}$, a disk, $|P| \leq 1$;

c) $M=S^{1} \times I$, an annulus, with $P=\emptyset$;

d) $M=T^{2}$ the torus, with $P=\emptyset$.

Recall that a subsurface $N \subset M$ is essential if no component of $\overline{M \backslash N}$ is a disk disjoint from $P$. If some component $N^{\prime}$ of $\overline{M \backslash N}$ is a disk with $\left|N^{\prime} \cap P\right|=1$, we call $N^{\prime}$ a pointed disk exterior to $N$.

Theorem 4.1 Consider an essential subsurface $N \subset M$ with the associated homomorphism induced by inclusion:

$$
i_{*}: \mathcal{M}(N, N \cap P) \rightarrow \mathcal{M}(M, P)
$$

i) If $N$ is a disk and $|N \cap P| \leq 1$ then $\mathcal{M}(N, N \cap P)$ is trivial, and therefore $i_{*}$ is injective.

ii) Suppose $N$ is an annulus and $N \cap P=\emptyset$. If $N$ has an exterior pointed disk, then the kernel of $i_{*}$ is $\mathcal{M}(N, N \cap P)$; otherwise $i_{*}$ is injective.

iii) Assuming that $(N, N \cap P)$ is not as in (i) or (ii), let $a_{1}, \ldots, a_{r}$ denote the boundary components of $N$ which bound pointed disks exterior to $N$, and let $b_{j}, b_{j}^{\prime}, j=1, \ldots, s$ be the pairs of boundary components of $N$ which cobound exterior cylinders (disjoint from P) (see Figure 9). Denote by $A_{i}, B_{j}, B_{j}^{\prime}$ the Dehn twists corresponding to the curves $a_{i}, b_{j}, b_{j}^{\prime}$, respectively. Then the kernel of $i_{*}$ is generated by

$$
\left\{A_{1}, \ldots, A_{r}, B_{1}^{-1} B_{1}^{\prime}, \ldots, B_{s}^{-1} B_{s}^{\prime}\right\}
$$

and is isomorphic to $\mathbf{Z}^{r+s}$. 


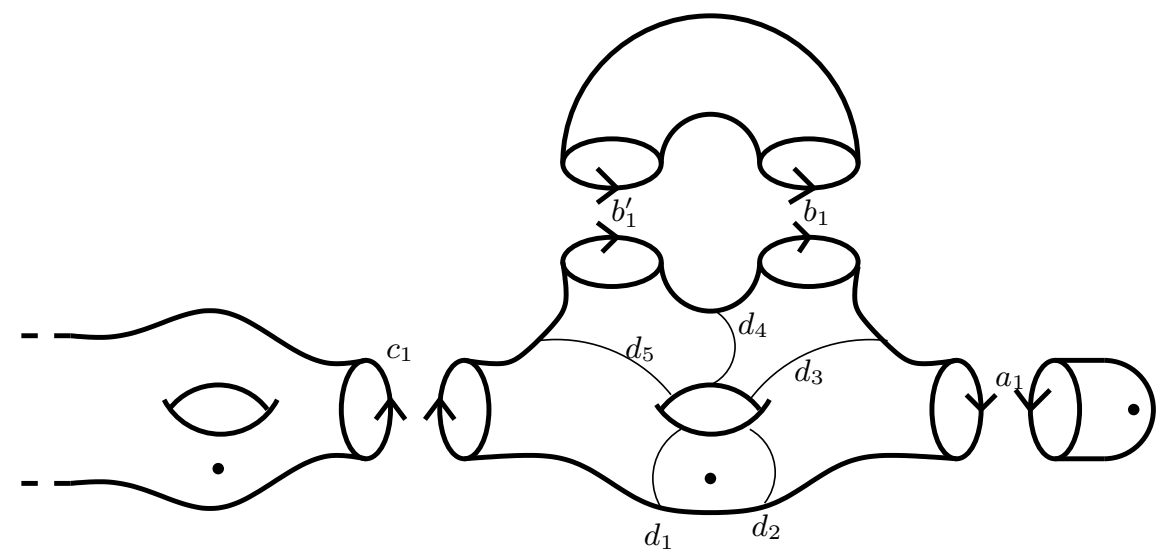

Figure 9: Subsurface $N$ and pantalon decomposition.

Proof: Part (i) is obvious and (ii) is a direct consequence of Proposition 3.8. To prove (iii), let $[h] \in \operatorname{Ker}\left(i_{*}\right)$, where $h: N \rightarrow N$. We have the following commutative diagram, with exact rows.

$$
\begin{aligned}
& 1 \rightarrow \mathcal{P} \mathcal{M}(N, P \cap N) \rightarrow \mathcal{M}(N, P \cap N) \quad \rightarrow \quad \Sigma_{P \cap N} \rightarrow 1 \\
& 1 \rightarrow \mathcal{P} \mathcal{M}(M, P) \quad \rightarrow \quad \mathcal{M}(M, P) \quad \rightarrow \quad \Sigma_{P} \rightarrow 1
\end{aligned}
$$

Since the homomorphism $\Sigma_{P \cap N} \rightarrow \Sigma_{P}$ is injective, $[h]$ is in $\mathcal{P} \mathcal{M}(N, P \cap N)$.

Let $c_{1}, \ldots, c_{t}$ denote the components of $\partial N$ different from the $a_{i}$ and $b_{j}, b_{j}^{\prime}$. In addition, let $d_{1}, \ldots, d_{u}: S^{1} \rightarrow N \backslash P$ be simple closed curves which determine a pantalon decomposition of $(N, N \cap P)$ (see Figure 9). Note that all the curves we are considering are pairwise disjoint and non-isotopic. Since $h$ is isotopic to the identity in $M \backslash P$, each $h \circ d_{i}$ is isotopic to $d_{i}$ in $M \backslash P$. Proposition 3.5 implies that $h \circ d_{i}$ is isotopic to $d_{i}$ in $N \backslash N \cap P$. By Proposition 3.10 we may suppose that $h \circ d_{i}=d_{i}$ for all $i=1, \ldots, u$, and that $h$ is the identity on the boundary of each pantalon. Using the structure of the pure mapping class groups of pantalons we conclude that

$$
[h]=A_{1}^{\alpha_{1}} \cdots A_{r}^{\alpha_{r}} B_{1}^{\beta_{1}} B_{1}^{\prime \beta_{1}^{\prime}} \cdots B_{s}^{\beta_{s}} B_{s}^{\prime \beta_{s}^{\prime}} C_{1}^{\gamma_{1}} \cdots C_{t}^{\gamma_{t}} D_{1}^{\delta_{1}} \cdots D_{u}^{\delta_{u}} .
$$

Therefore

$$
1=i_{*}[h]=B_{1}^{\beta_{1}+\beta_{1}^{\prime}} \cdots B_{s}^{\beta_{s}+\beta_{s}^{\prime}} C_{1}^{\gamma_{1}} \cdots C_{t}^{\gamma_{t}} D_{1}^{\delta_{1}} \cdots D_{u}^{\delta_{u}} .
$$

By Proposition 3.8

$$
\beta_{1}+\beta_{1}^{\prime}=\cdots=\beta_{s}+\beta_{s}^{\prime}=\gamma_{1}=\cdots=\gamma_{t}=\delta_{1}=\cdots=\delta_{u}=0 .
$$


Therefore

$$
[h]=A_{1}^{\alpha_{1}} \cdots A_{r}^{\alpha_{r}}\left(B_{1}^{-1} B_{1}^{\prime}\right)^{\beta_{1}^{\prime}} \cdots\left(B_{s}^{-1} B_{s}^{\prime}\right)^{\beta_{s}^{\prime}} .
$$

Conversely, it is clear that any $[h]$ of this form is in the kernel of $i_{*}$. Finally, Proposition 3.8 implies that $\operatorname{Ker}\left(i_{*}\right)$ is isomorphic to $\mathbf{Z}^{r+s}$.

Corollary 4.2 Let $N \subset M$ be any subsurface (with $\partial N \cap P=\emptyset$ ) and let

$$
i_{*}: \mathcal{M}(N, N \cap P) \rightarrow \mathcal{M}(M, P)
$$

the natural homomorphism.

i) If $N$ is a disk and $|N \cap P| \leq 1$, then $i_{*}$ is injective.

ii) If $N$ is an annulus and $N \cap P=\emptyset$, then $i_{*}$ is injective if and only if there is no boundary component of $N$ which is the boundary of a disk intersecting $P$ in less than two points.

iii) If $(N, N \cap P)$ is not as in (i) or (ii), then $i_{*}$ is injective if and only if no component of $\overline{M \backslash N}$ is either an annulus disjoint from $P$ whose boundary components are both boundary components of $N$, or a disk which contains less than two points of $P$.

\section{Centres}

We begin this section by considering a special mapping of the surface $M$ of genus one, and with one boundary component; that is, $M$ is a torus minus a disk, with empty puncture set $P$. We model $M$ as a certain identification space of a planar surface, as follows (see Figure 10). Let

$$
\begin{gathered}
D=\{z \in \mathbf{C} ;|z| \leq 4\} \\
D_{1}=\{z \in \mathbf{C} ;|z-2|<1\} \\
D_{2}=\{z \in \mathbf{C} ;|z+2|<1\}
\end{gathered}
$$

Then $D \backslash\left(D_{1} \cup D_{2}\right)$ is a pantalon (of type III) with boundary curves $a_{1}, a_{2}, c$ : $S^{1} \rightarrow \partial M$ which we parametrize as follows, $0 \leq \theta \leq 2 \pi$ :

$$
\begin{gathered}
c\left(e^{i \theta}\right)=4 e^{i \theta} \\
a_{1}\left(e^{i \theta}\right)=2+e^{i \theta} \\
a_{2}\left(e^{i \theta}\right)=-2-e^{-i \theta} .
\end{gathered}
$$




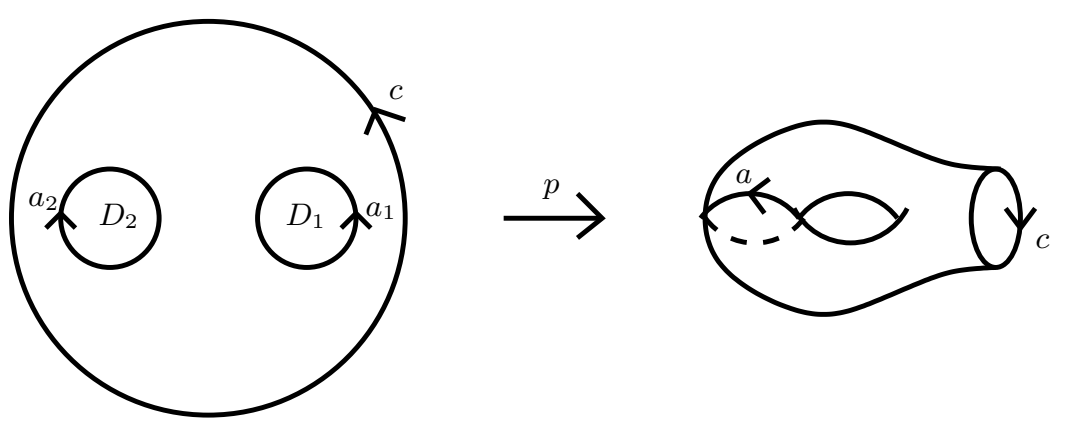

Figure 10: The projection map.

We consider $M=\left(D \backslash\left(D_{1} \cup D_{2}\right)\right) / \sim$ where we identify the points on the curves $a_{1}$ and $a_{2}$ by

$$
a_{1}\left(e^{i \theta}\right) \sim a_{2}\left(e^{i \theta}\right)
$$

Denote the natural projection by

$$
p: D \backslash\left(D_{1} \cup D_{2}\right) \rightarrow M .
$$

The "meridian" curve $a: S^{1} \rightarrow M$ is defined by

$$
a=p \circ a_{1}=p \circ a_{2} .
$$

Now we define a homeomorphism $R: D \rightarrow D$ by the equation:

$$
R\left(r e^{i \theta}\right)= \begin{cases}r e^{i(\theta-\pi)} & \text { if } 0 \leq r \leq 3 \\ r e^{i(\theta-(r-2) \pi)} & \text { if } 3 \leq r \leq 4\end{cases}
$$

We have $R\left(D_{1}\right)=D_{2}, \quad R\left(D_{2}\right)=D_{1}, \quad R \circ a_{1}=a_{2}^{-1}$ and $R \circ a_{2}=a_{1}^{-1}$. Therefore, $R$ induces a homeomorphism

$$
\tilde{R}: M \rightarrow M
$$

such that

$$
\tilde{R} \circ a=a^{-1} .
$$

Its class $\rho=[\tilde{R}] \in \mathcal{M}(M)$ will be called a half-twist of $M$ along $c$ relative to $a$. The Dehn twists $A$ and $C$ about the respective curves $a$ and $c$ relate to $\rho$ as follows:

$$
\rho^{2}=C, \quad \rho A \rho^{-1}=A .
$$

The latter equation follows since $\rho A \rho^{-1}$ is the Dehn twist about $\rho \circ a=a^{-1}$, and the Dehn twist about $a^{-1}$ is the same as the Dehn twist about $a$ for any simple closed curve $a$. 
Proposition 5.1 Let $M, \rho, a$ and $A$ be as in the above discussion. If $G$ is the subgroup of $\mathcal{M}(M)$ consisting of classes of homomorphisms $h: M \rightarrow M$ such that $h \circ a$ is isotopic with $a$ or $a^{-1}$, then $G$ is generated by $\{A, \rho\}$ and is isomorphic with $\mathbf{Z}^{2}$.

Proof: Let $[h] \in G$; we may assume $h \circ a=a$ or $h \circ a=a^{-1}$. Note that $a$ determines a pantalon decomposition of $M$, with a single pantalon of type III, namely $D \backslash\left(D_{1} \cup D_{2}\right)$.

If $h \circ a=a$, by the structure of the mapping class group of the pantalon, for some integers $m, n$ :

$$
[h]=A^{n} C^{m}=A^{n} \rho^{2 m} .
$$

If $h \circ a=a^{-1}$, then $\tilde{R} \circ h \circ a=a$ and $\rho[h]$ has the form

$$
\rho[h]=A^{n} C^{m}=A^{n} \rho^{2 m} .
$$

Therefore

$$
[h]=\rho^{-1} A^{n} \rho^{2 m}=A^{n} \rho^{2 m-1} .
$$

It is clear that $A$ and $\rho$ are in $G$, so they generate $G$ and we have already noted that they commute. If $A^{n} \rho^{m}=1$, then

$$
\left(A^{n} \rho^{m}\right)^{2}=A^{2 n} \rho^{2 m}=A^{2 n} C^{m}=1,
$$

and by Proposition $3.8,2 n=m=0$. This shows $G \cong \mathbf{Z}^{2}$.

We turn now to determining the centre $\mathcal{Z} \mathcal{M}(M, P)$ of an arbitrary (compact orientable) surface $M$ with puncture set $P$, that is, the subgroup of $\mathcal{M}(M, P)$ consisting of mapping classes which commute with all elements of $\mathcal{M}(M, P)$. First we record some simple cases: If $M$ is a sphere or disk and $|P| \leq 1, \mathcal{Z M}(M, P)=$ $\mathcal{M}(M, P)=\{1\} \cdot \mathcal{Z} \mathcal{M}\left(S^{2}, 2\right)=\mathcal{M}\left(S^{2}, 2\right)=\mathbf{Z} / 2 \mathbf{Z} \cdot \mathcal{M}\left(S^{2}, 3\right) \cong \Sigma_{3}$ and therefore $\mathcal{Z M}\left(S^{2}, 3\right)$ is the trivial group. Each of the pantalons (type I, II or III) has an abelian mapping class group, so the centre is equal to the whole group. For the same reason $\mathcal{Z} \mathcal{M}\left(S^{1} \times I\right)=\mathcal{M}\left(S^{1} \times I\right) \cong \mathbf{Z}$.

The case of the torus is somewhat more interesting. Since

$$
\mathcal{M}\left(T^{2}, 0\right) \cong \mathcal{M}\left(T^{2}, 1\right) \cong S L(2, \mathbf{Z})
$$

we see algebraically that the centre is the cyclic group of order two, consisting of the two diagonal matrices, $\pm I$, where $I$ is the identity matrix. However, to warm up for the more complicated cases, whe will establish this fact geometrically. Consider the torus $T^{2}$ embedded in $x y z$-space as the set of points of distance 1 from 
the circle $x^{2}+y^{2}=4, z=0$ (see Figure 11). Let $s: T^{2} \rightarrow T^{2}$ be the (orientationpreserving) involution $s(x, y, z)=(x,-y,-z)$. In the case of $\mathcal{M}\left(T^{2}, 1\right)$, we suppose that $P=\{p\}$ is a point on the $x$-axis, so that $s(p)=p$. Let $\sigma=[s] \in \mathcal{M}\left(T^{2}\right)$ and $\sigma_{1}=[s] \in \mathcal{M}\left(T^{2},\{p\}\right)$.

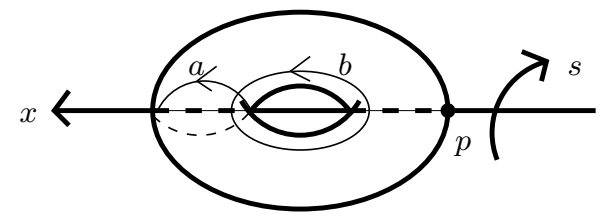

Figure 11: Involution generating the centre of $\mathcal{M}\left(T^{2}\right)$.

Proposition 5.2 The centre of $\mathcal{M}\left(T^{2}\right)$ is the cyclic group of order 2 generated by $\sigma$; similarly $\mathcal{Z} \mathcal{M}\left(T^{2}, 1\right) \cong \mathbf{Z} / 2 \mathbf{Z}$, generated by $\sigma_{1}$.

Proof: We prove the first part; the case of $\mathcal{Z M}\left(T^{2},\{p\}\right)$ being essentially the same. Since $s$ has order $2, \sigma^{2}=1$ in $\mathcal{M}\left(T^{2}\right)$. Let $a, b: S^{1} \rightarrow T^{2}$ be the circles parametrized by

$$
a\left(e^{i \theta}\right)=(2+\cos \theta, 0, \sin \theta), \quad b\left(e^{i \theta}\right)=(\cos \theta, \sin \theta, 0) .
$$

Then

$$
s \circ a=a^{-1} \text { and } s \circ b=b^{-1} .
$$

Since $a$ is not isotopic to $a^{-1}$ we conclude that $\sigma \neq 1$, and so $\sigma$ has order 2. Letting $A, B$ be the Dehn twists about $a, b$ we have

$$
\sigma A \sigma^{-1}=A \text { and } \sigma B \sigma^{-1}=B
$$

and since $A$ and $B$ generate $\mathcal{M}\left(T^{2}\right)$ we conclude that $\sigma \in \mathcal{Z M}\left(T^{2}\right)$.

Now suppose that $[h] \in \mathcal{Z} \mathcal{M}\left(T^{2}\right)$, where $h: T^{2} \rightarrow T^{2}$. Then $A=[h] A[h]^{-1}=C$ is a Dehn twist about the curve $c=h \circ a$. By Proposition 3.6, $c \simeq a^{ \pm 1}$, so we may assume that $h \circ a=a$ or $h \circ a=a^{-1}$. Case $1, h \circ a=a$; we can cut $T^{2}$ open along $a$ and conclude from the mapping class of the cylinder that $[h]=A^{k}$ for some integer $k$. Since $I(a, b)=1$ and $A^{k}$ and $B$ commute, Proposition 3.7 implies that $k=0$, and $[h]=1$ in this case. Case 2, $h \circ a=a^{-1}$. Then $s \circ h \circ a=a$ and we conclude from case 1 that $\sigma[h]=1$, so $[h]=\sigma^{-1}=\sigma$.

Next we consider the torus with two marked points. Using the above model for $T^{2}$, let $P=\{(0,3,0),(0,-3,0)\}$, that it, a pair of points which are interchanged 
by the involution $s: T^{2} \rightarrow T^{2}$ described above. Let $\sigma_{2} \in \mathcal{M}\left(T^{2}, P\right)=\mathcal{M}\left(T^{2}, 2\right)$ be the class represented by $s$. The following is proved in the same manner as the above.

Proposition 5.3 The centre of $\mathcal{M}\left(T^{2}, 2\right)$ is the cyclic group of order 2 generated by $\sigma_{2}$.

We now consider the closed surface $M$ of genus 2 , which we realize in $\mathbf{R}^{3}$ as the boundary of a uniform regular neighborhood of the union of the circles

$$
(x+1)^{2}+y^{2}=1, z=0 \text { and }(x-1)^{2}+y^{2}=1, z=0 .
$$

Let $s: \mathbf{R}^{3} \rightarrow \mathbf{R}^{3}$ be the same involution as in the previous discussion, and let $\sigma \in \mathcal{M}(M)$ be represented by the restriction of $s$ to $M$.

Proposition 5.4 The centre of $\mathcal{M}(M)$, where $M$ is a closed surface of genus 2, is the cyclic group of order 2 generated by $\sigma$.

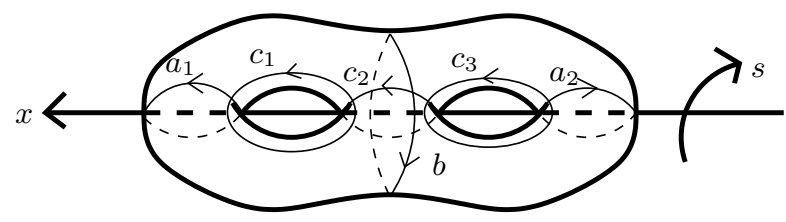

Figure 12: Generator of the centre of $\mathcal{M}\left(M_{2}\right)$.

Proof: Let $a_{1}, a_{2}, b, c_{1}, c_{2}, c_{3}$ be the simple closed curves as in Figure 12. In particular, $b$ is the curve of intersection of $M$ with the plane $x=0, c_{1}$ and $c_{3}$ lie in the plane $z=0$ and $a_{1}, a_{2}, c_{2}$ are in the plane $y=0$. We have

$$
s \circ a_{i}=a_{i}^{-1}, \quad s \circ b=b, \quad s \circ c_{i}=c_{i}^{-1} .
$$

Since $a_{1}$ is not isotopic with $a_{1}^{-1}, \sigma \neq 1$ has order 2 in $\mathcal{M}(M)$. Let $A_{1}, A_{2}, B, C_{1}$, $C_{2}, C_{3}$ be the Dehn twists about the respective curves, which generate $\mathcal{M}(M)$, according to 23]. From the above equations we conclude that

$$
\sigma A_{i} \sigma^{-1}=A_{i}, \quad \sigma B \sigma^{-1}=B, \quad \sigma C_{i} \sigma^{-1}=C_{i},
$$

so $\sigma$ is indeed central in $\mathcal{M}(M)$.

Given $[h] \in \mathcal{Z} \mathcal{M}(M)$ we have $A_{i}=[h] A_{i}[h]^{-1}$, which is a Dehn twist about $h \circ a_{i}$. By Proposition $3.6 h \circ a_{i}$ is isotopic with $a_{i}^{ \pm 1}$. Similarly $h \circ b$ is isotopic with 
$b^{ \pm 1}$. By Proposition 3.10 we may assume that $h \circ a_{i}=a_{i}$ or $a_{i}^{-1}$ and that $h \circ b=b$ or $b^{-1}$.

Let $M_{1}$ and $M_{2}$ be the closures of the two components of $M \backslash b$, with $a_{1}$ in $M_{1}$, $a_{2}$ in $M_{2}$. Now $h \circ b=b^{-1}$ can only happen if $h\left(M_{1}\right)=M_{2}$ and $h\left(M_{2}\right)=M_{1}$, which is impossible because $h \circ a_{i}=a_{i}^{ \pm 1}$. So we must have $h \circ b=b, h\left(M_{i}\right)=M_{i}$. Letting $\rho_{i}$ denote the half-twist of $M_{i}$ along $b$ relative to $a_{i}$, Proposition 5.1 implies that $[h]$ can be written in the form

$$
[h]=\rho_{1}^{n_{1}} A_{1}^{m_{1}} \rho_{2}^{n_{2}} A_{2}^{m_{2}},
$$

for some integers $n_{1}, n_{2}, m_{1}, m_{2}$. Consider

$$
[h]^{2}=A_{1}^{2 m_{1}} A_{2}^{2 m_{2}} B^{n_{1}+n_{2}} .
$$

Since $[h]^{2}$ is central it commutes with $C_{1}$, as do $A_{2}$ and $B$. Therefore $A_{1}^{2 m_{1}}$ also commutes with $C_{1}$. But $I\left(a_{1}, c_{1}\right)=1$, so by Proposition 3.7, $2 m_{1}=0$. Similarly $2 m_{2}=0$ and $n_{1}+n_{2}=0$. We note that either $n_{1}$ and $n_{2}$ are both odd or both even. If they are both even, then $h \circ a_{1}=a_{1}$ and $h \circ a_{2}=a_{2}$, if both odd, then $h \circ a_{1}=a_{1}^{-1}$ and $h \circ a_{2}=a_{2}^{-1}$.

Case $1, n_{1}$ and $n_{2}$ are even: write $n_{1}=2 k_{1}$ and $n_{2}=2 k_{2}$. Then

$$
[h]=\rho_{1}^{2 k_{1}} \rho_{2}^{2 k_{2}}=B^{k_{1}+k_{2}}=B^{0}=1,
$$

because $k_{1}+k_{2}=\left(n_{1}+n_{2}\right) / 2=0$.

Case $2, n_{1}$ and $n_{2}$ are odd. Then

$$
s \circ h \circ a_{1}=a_{1} \text { and } s \circ h \circ a_{2}=a_{2}
$$

and by case $1, \sigma[h]=1$. Therefore $[h]=\sigma^{-1}=\sigma$.

Proposition 5.5 Let $M$ denote the genus one oriented surface with one boundary component. Then the centre of $\mathcal{M}(M)$ is the infinite cyclic group generated by the half-twist $\rho$, defined in the discussion preceding Proposition 5.1.

Proof: Let $a$ be the curve on $M, A$ the Dehn twist about $a, \tilde{R}: M \rightarrow M, \rho=[\tilde{R}]$, all as described in the discussion preceding Proposition 5.1. Let $b$ be the curve on $M$ which is the image, under the identification $D \backslash\left(D_{1} \cup D_{2}\right) \rightarrow M$, of the interval $[-1,1]$, so that $I(a, b)=1$. Let $B$ be the Dehn twist about $b$; then $\{A, B\}$ generates $\mathcal{M}(M)$.

Noting that $\tilde{R} \circ a=a^{-1}$ and $\tilde{R} \circ b=b^{-1}$, we see that $\rho$ commutes with $A$ and $B$, and therefore $\rho$ is in the centre of $\mathcal{M}(M)$. Since $\rho^{2}=C$ and $C$ has infinite order, $\rho$ has also infinite order. 
Now let $\xi$ be in the centre of $\mathcal{M}(M)$, and $h: M \rightarrow M$ a homeomorphism with $[h]=\xi$. Then $A=\xi A \xi^{-1}$ is a Dehn twist about $h \circ a$, so by Proposition $3.6 h \circ a$ is isotopic with $a$ or $a^{-1}$. By Proposition $5.1 \xi$ is of the form

$$
\xi=A^{p} \rho^{q}, \quad p, q \in \mathbf{Z} .
$$

Because $\xi$ and $\rho$ commute with $B, A^{p}$ commutes with $B$. But $I(a, b)=1$, so Proposition 3.7 implies that $p=0$ and therefore $\xi=\rho^{q}$.

Remark: Let $M$ be a genus one oriented surface with one boundary component as above. According to [36] $\mathcal{M}(M)$ is isomorphic to the Artin braid group $\langle A, B \mid A B A=B A B\rangle$, by [4] its center is the infinite cyclic group generated by $(A B A)^{2}$, and one can check directly that $\rho=(A B A)^{2}$.

Following is the main result regarding the centre of $\mathcal{M}(M, P)$, for a general Riemann surface. We have already noted that there are certain exceptional cases, so we list the hypotheses here for the generic result:

1) If $M$ is a sphere, then assume $|P| \geq 4$,

2) If $M$ is a disk, then assume $|P| \geq 3$,

3) If $M$ is an annulus, then assume $|P| \geq 1$,

4) If $M$ is a torus, then assume $|P| \geq 3$,

5) If $M$ is a surface of genus one and one boundary component, then assume $|P| \geq 1$,

6) If $M$ is a surface of genus 2 , then assume $|P| \geq 1$.

Theorem 5.6 Let $M$ be a connected compact orientable surface with marked points $P \subset M$ and assume the hypotheses (1) - (6) above. Let $c_{1}, \cdots, c_{q}: S^{1} \rightarrow \partial M$ be the boundary curves of $M$ and $C_{1}, \cdots, C_{q}$ the Dehn twists about these curves. Then the centre $\mathcal{Z} \mathcal{M}(M, P)$ of $\mathcal{M}(M, P)$ is the subgroup generated by $\left\{C_{1} \cdots, C_{q}\right\}$ and is isomorphic with $\mathbf{Z}^{q}$. In particular, if $\partial M$ is empty, the centre of $\mathcal{M}(M, P)$ is trivial.

Proof: It is clear that $C_{i}$ is central in $\mathcal{M}(M, P)$; the fact that the subgroup generated by $C_{1}, \cdots, C_{q}$ is isomorphic with $\mathbf{Z}^{q}$ follows directly from Proposition 3.8. Now consider an element $\xi$ in the centre of $\mathcal{M}(M, P)$, with representative homeomorphism $h: M \rightarrow M, \quad[h]=\xi$.

Consider curves $a_{1}, \cdots, a_{p}$ which detemine a pantalon decomposition of $(M, P)$. (All the cases which do not admit a pantalon decomposition have been excluded.) Since $\xi$ is central, the Dehn twist $A_{i}$ about the curves $a_{i}$ satisfy

$$
A_{i}=\xi A_{i} \xi^{-1},
$$


and since $\xi A_{i} \xi^{-1}$ is a Dehn twist about $h \circ a_{i}$ we conclude from Proposition 3.6 that $h \circ a_{i}$ is isotopic with $a_{i}$ or $a_{i}^{-1}$. By Proposition 3.10 we may suppose that

$$
h \circ a_{i}=a_{i}^{ \pm 1},
$$

and consequently, $h$ permutes the pantalons.

The pantalons $\left(M_{j}, P_{j}\right)$ are mapped to $M$ by maps $\phi_{j}$. We call a curve $a_{i}$ separating if it is in the image of $\phi_{k}$ and $\phi_{l}$, for some $k \neq l$ (a separating curve in our sense need not separate the surface itself). First assume that $a_{i}$ is separating and that $h \circ a_{i}=a_{i}^{-1}$. Then $h\left(\phi_{k}\left(M_{k}\right)\right)=\phi_{l}\left(M_{l}\right)$ and $h\left(\phi_{l}\left(M_{l}\right)\right)=\phi_{k}\left(M_{k}\right)$. We consider all the possibilities:

If $\left(M_{k}, P_{k}\right)$ and $\left(M_{l}, P_{l}\right)$ are pantalons of type I, then $M$ is a 2 -sphere and $|P|=4$ (see Figure 13.i). Consider the exact sequence:

$$
1 \rightarrow \mathcal{P} \mathcal{M}\left(S^{2}, 4\right) \rightarrow \mathcal{M}\left(S^{2}, 4\right) \rightarrow \Sigma_{4} \rightarrow 1 .
$$

Since the centre of $\Sigma_{4}$ is trivial, $\xi$ must act trivially on $P$, whereas $h$ interchanges the points $\phi_{k}\left(P_{k}\right)$ and $\phi_{l}\left(P_{l}\right)$, so this case cannot occur.

If $\left(M_{k}, P_{k}\right)$ and $\left(M_{l}, P_{l}\right)$ are pantalons of type II, then $\phi_{k}\left(M_{k}\right) \cap \phi_{l}\left(M_{l}\right)=a_{i}$ and there are other curves $a_{\mu}$ and $a_{\nu}$ which form the remaining boundary curves of these pantalons (see Figure 13. ii). Then, since $h$ interchanges the pantalons, $h\left(a_{\mu}\right)=a_{\nu}^{ \pm 1}$. On the other hand, $h\left(a_{\mu}\right)=a_{\mu}^{ \pm 1}$, so we must have $a_{\mu}=a_{\nu}^{ \pm 1}$ and conclude that $M$ is a torus and $|P|=2$. This case has been excluded.

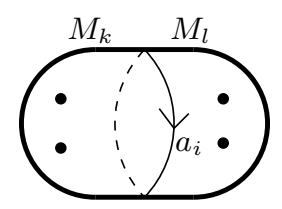

(i)

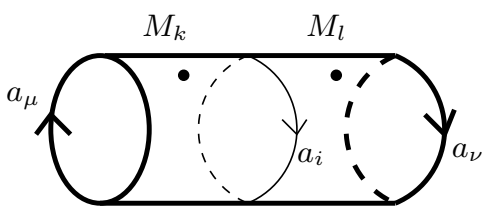

(ii)

Figure 13: First cases, $a_{i}$ separating.

If $\left(M_{k}, P_{k}\right)$ and $\left(M_{l}, P_{l}\right)$ are pantalons of type III with identifications, so that $\phi_{k}\left(M_{k}\right)$ and $\phi_{l}\left(M_{l}\right)$ are genus 1 surfaces with one boundary component $a_{i}$, then $M$ is a closed surface of genus 2 and $P$ is empty (see Figure 14.i). This case also has been excluded.

Finally suppose $\left(M_{k}, P_{k}\right)$ and $\left(M_{l}, P_{l}\right)$ are pantalons of type III, mapped homeomorphically by $\phi_{k}$ and $\phi_{l}$ (see Figure 14.ii). Then we argue as in the type II case that their other boundary components must be identified, and that $M$ is closed, has genus 2 , and $P$ is empty, an excluded case. 


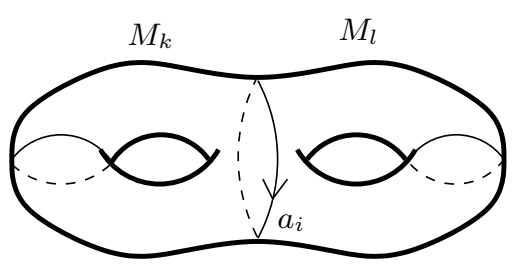

(i)

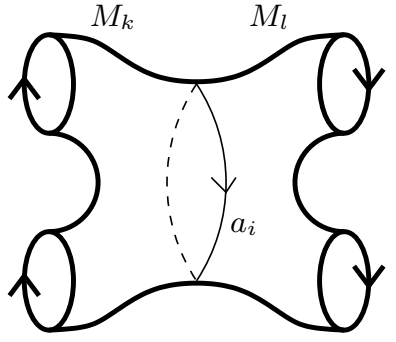

(ii)

Figure 14: $a_{i}$ separating type III pantalons.

Thus we have shown that if $a_{i}$ is separating, then $h \circ a_{i}=a_{i}$. In addition we have seen that $h\left(\phi_{k}\left(M_{k}\right)\right)=\phi_{k}\left(M_{k}\right)$ for all $k$.

Recall the notation $m=|P|$ and $q$ is the number of boundary components of $M$. We complete the proof by considering three cases:

Case 1: $m+q \geq 2$. Then we may assume all the $a_{i}$ are separating (see Figure 15). If $M$ is not a sphere or disk, then each pantalon can be taken to be of type II or III. If $M$ is either a sphere or disk, the exact sequence

$$
1 \rightarrow \mathcal{P} \mathcal{M}(M, P) \rightarrow \mathcal{M}(M, P) \rightarrow \Sigma_{P} \rightarrow 1,
$$

and the fact that $\Sigma_{P}$ has trivial centre under the assumption $|P| \geq 3$, shows that $\xi \in \mathcal{P} \mathcal{M}(M, P)$, or in other words, $h$ fixes $P$ pointwise. So we have, by the structure of the (pure) mapping class groups of the pantalons of type I, II and III:

$$
\xi=A_{1}^{r_{1}} \cdots A_{p}^{r_{p}} C_{1}^{s_{1}} \cdots C_{q}^{s_{q}},
$$

for some integers $r_{1}, \ldots, r_{p}, s_{1}, \ldots, s_{q}$. Now fix $i \in\{1, \ldots, p\}$. By Proposition 3.4, there exists a generic simple closed curve $b: S^{1} \rightarrow M \backslash P$ such that $I\left(a_{i}, b\right)>0$ but $a_{j} \cap b=\emptyset$ if $j \neq i$. If $B$ is the Dehn twist about $b$, we see that $B$ commutes with $A_{j}, j \neq i$. $B$ also commutes with all the $C_{j}$ and with $\xi$ since they are central. It follows that $B$ commutes with $A_{i}^{r_{i}}$. By Proposition 3.7 we conclude that $r_{i}=0$. Since $i$ was arbitrary,

$$
\xi=C_{1}^{s_{1}} \cdots C_{q}^{s_{q}}
$$

Case 2: $m+q=1$. We may now suppose that $a_{1}$ is not separating, but $a_{2}, \ldots, a_{p}$ are separating curves, and the pantalon $\left(M_{1}, P_{1}\right)$ is of type III with two boundary curves identified to $a_{1}$, so that $\phi_{1}\left(M_{1}\right)$ is a surface of genus one with one boundary component, which we may take to be $a_{2}$ (see Figure 16). Moreover we 


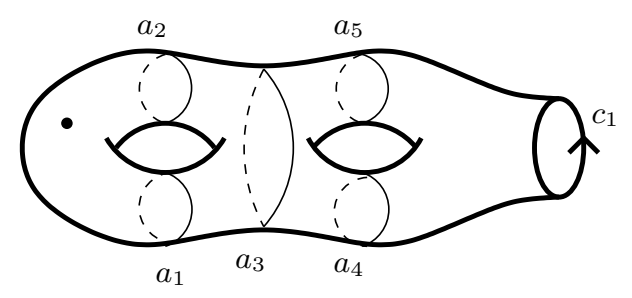

Figure 15: Pantalon decomposition, all $a_{i}$ separating.

may assume $\left(M_{2}, P_{2}\right), \ldots,\left(M_{r}, P_{r}\right)$ are pantalons of type II or III, embedded in $M$. Let $\rho$ denote the half-twist of $\phi_{1}\left(M_{1}\right)$ along $a_{2}$ relative to $a_{1}$. Then by Proposition 5.1 and the structure of the mapping class groups of type II and III pantalons, $\xi$ can be written (assuming $q=1, m=0$ and noting that $\rho^{2}=A_{2}$ ):

$$
\xi=\rho^{k} A_{1}^{r_{1}} A_{3}^{r_{3}} \cdots A_{p}^{r_{p}} C_{1}^{s_{1}}
$$

for integers $k, r_{1}, r_{3}, \ldots, r_{p}, s_{1}$. Then

$$
\xi^{2}=A_{1}^{2 r_{1}} A_{2}^{k} A_{3}^{2 r_{3}} \cdots A_{p}^{2 r_{p}} C_{1}^{2 s_{1}} .
$$

Employing the same argument as in Case 1, we conclude that

$$
2 r_{1}=k=2 r_{3}=\cdots=2 r_{p}=0
$$

and so $\xi=C_{1}^{s_{1}}$ if $q=1$. If $q=0$ and $m=1$ we similarly conclude that $\xi=1$.

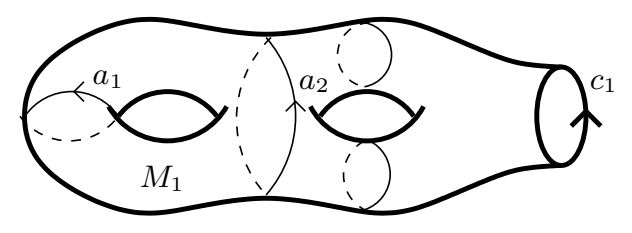

Figure 16: Pantalon decomposition with $a_{1}$ nonseparating.

Case 3: $m+q=0$. In particular, $q=0$. Then we need two singular pantalons in the decomposition of $M, \xi$ has an expression as a product of two half-twists together with powers of the $A_{i}$ and we conclude by examining $\xi^{2}$, exactly as in case 2 that the powers are all zero and therefore $\xi=1$. 


\section{Commensurability}

If $G$ is a group, then two subgroups $H, H^{\prime}<G$ are said to be commensurable if $H \cap H^{\prime}$ has finite index in both $H$ and $H^{\prime}$. Commensurability is an equivalence relation on the set of all subgroups of $G$, really of interest only for infinite groups. Following is an elementary property of commensurable subgroups, which we shall find useful.

Proposition 6.1 Suppose $H$ and $H^{\prime}$ are commensurable subgroups of $G$. Then for each $h \in H$, there exists a nonzero integer $k$ such that $h^{k} \in H^{\prime}$.

Proof: If not, then $\left\{h^{k}\right\}, k \in \mathbf{Z}$ is an infinite set of elements, all in different cosets of $H$ rel $H \cap H^{\prime}$, contradicting finite index.

Proposition 6.2 Suppose $a_{1}, \ldots, a_{p}: S^{1} \rightarrow M \backslash P$ are essential simple closed curves which are pairwise disjoint. Let $b: S^{1} \rightarrow M \backslash P$ be an essential simple closed curve such that $I\left(a_{i}, b\right)=0$ for all $i=1, \ldots, p$. Then there exists a simple closed curve $c: S^{1} \rightarrow M \backslash P$ isotopic to $b$ and such that $a_{i} \cap c=\emptyset$ for all $i=1, \ldots, p$.

Proof: We may assume $b$ transverse to all the $a_{i}$ and argue by induction on the cardinality of $b \cap\left(a_{1} \cup \cdots \cup a_{p}\right)$. If $b \cap\left(a_{1} \cup \cdots \cup a_{p}\right)$ is empty, there is nothing to prove; suppose it is nonempty. Choose $i$ so that $b \cap a_{i} \neq \emptyset$. Proposition 3.2 implies that $b$ and $a_{i}$ cobound a bigon $D$. There may be other intersections of $a_{j}$ with $D$, but there is always an outermost bigon $D^{\prime} \subset D$ cobounded by $b$ and some $a_{k}$, and otherwise disjoint from $a_{1} \cup \cdots \cup a_{p}$ (see Figure 17). Now we can push $b$ across $D^{\prime}$ to obtain a curve $b^{\prime}$ isotopic with $b$ and

$$
\left|b^{\prime} \cap\left(a_{1} \cup \cdots \cup a_{p}\right)\right|=\left|b \cap\left(a_{1} \cup \cdots \cup a_{p}\right)\right|-2 .
$$

By inductive hypothesis, there exists an isotopy from $b^{\prime}$ to an essential simple closed curve $c$ in $M \backslash P$ such that $a_{i} \cap c=\emptyset$ for all $i=1, \ldots, p$.

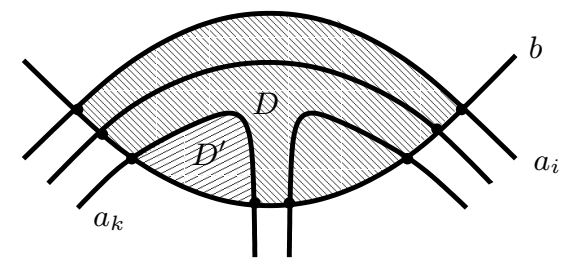

Figure 17: Bigon in proof of Proposition 6.2. 
Definitions: Recall that a subsurface is essential if none of its exterior components is a disk with zero marked points. By a marked subsurface we mean a pair $(N, Q)$ where $N$ is a subsurface of $M$ and $Q=P \cap N$. We will say that an essential marked subsurface $(N, Q)$ of $(M, P)$ is injective provided it satisfies:

a) If $N$ is a disk, then $|Q| \geq 2$,

b) If $N$ is an annulus with $Q$ empty, then there is no pointed disk component of the exterior of $N$ in $M$,

c) If $N$ is not an annulus, or if $|Q| \geq 1$, then no component of $\overline{M \backslash N}$ is a disk with one marked point, or a cylinder with no marked points and both boundary components in $\partial N$.

By Corollary 4.2 these criteria assure the injectivity of

$$
\mathcal{M}(N, Q) \rightarrow \mathcal{M}(M, P) .
$$

If $(N, Q)$ and $\left(N^{\prime}, Q^{\prime}\right)$ are marked subsurfaces of $(M, P)$, we say they are isotopic provided there is a continuous family of homeomorphisms $h_{t} \in \mathcal{H}(M, P), \quad t \in$ $[0,1]$ such that $h_{0}=$ identity, and $h_{1}(N, Q)=\left(N^{\prime}, Q^{\prime}\right)$. In particular, $Q=Q^{\prime}$.

We would like to be able to say that geometric subgroups are commensurable if and only if they are equal, if and only if their defining subsurfaces are isotopic. However, just as with centres, there are some exceptions to the general principle. Our first family are the infinite cyclic geometric subgroups. The only marked surfaces with mapping class $\mathbf{Z}$ are $\left(S^{1} \times I, 0\right)$ and $\left(D^{2}, 2\right)$; we note for future reference that these are the only mapping class groups which contain $\mathbf{Z}$ as a finiteindex subgroup.

Proposition 6.3 Suppose $(N, Q)$ and $\left(N^{\prime}, Q^{\prime}\right)$ are injective subsurfaces of $(M, P)$, and suppose $N=S^{1} \times I$ and $Q$ is empty. Suppose $\mathcal{M}(N, Q)$ and $\mathcal{M}\left(N^{\prime}, Q^{\prime}\right)$ are commensurable subgroups of $\mathcal{M}(M, P)$. Then either (1) $N^{\prime}$ is a cylinder $S^{1} \times I$ with $Q^{\prime}$ empty or (2) $N^{\prime}$ is a disk and $\left|Q^{\prime}\right|=2$.

In case (1), $N^{\prime}$ is isotopic with $N$. In case (2), $\left(N^{\prime}, Q^{\prime}\right)=\left(D^{2}, 2\right)$, then one of the components $\left(N_{1}, Q_{1}\right)$ of the exterior of $(N, Q)$ in $(M, P)$ is a $\left(D^{2}, 2\right)$, and $\left(N_{1}, Q_{1}\right)$ is isotopic with $\left(N^{\prime}, Q^{\prime}\right)$ (see Figure 18$)$.

Proof: Since $\mathcal{M}(N, Q)$ is infinite cyclic, so is every nontrivial subgroup, including $\mathcal{M}(N, Q) \cap \mathcal{M}\left(N^{\prime}, Q^{\prime}\right)$, so $\left(N^{\prime}, Q^{\prime}\right)$, its mapping class group containing a finite index $\mathbf{Z}$, can only be $\left(S^{1} \times I, \emptyset\right)$ or $\left(D^{2}, 2\right)$.

Case 1: $\left(N^{\prime}, Q^{\prime}\right)$ is $\left(S^{1} \times I, \emptyset\right)$. Let $a^{\prime}(z)=(z, 1 / 2), \quad z \in S^{1}$ denote the central curve of $N^{\prime}$ and similarly label the central curve of $N$ as $a: S^{1} \rightarrow N$, and let $A^{\prime}, A$ be Dehn twists of $M$ about these curves. $A$ and $A^{\prime}$ represent generators of 


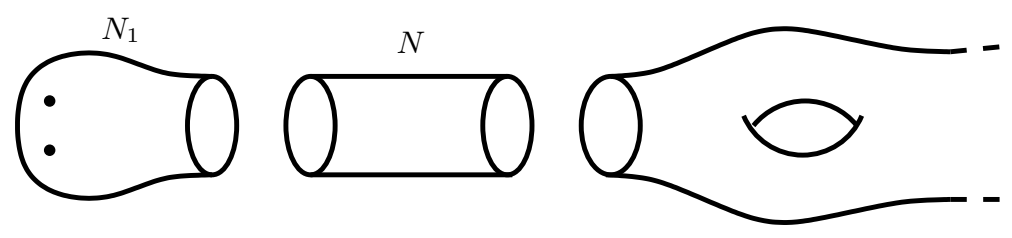

Figure 18: Injective subsurfaces in Proposition 6.3.

$\mathcal{M}(N, Q)$ and $\mathcal{M}\left(N^{\prime}, Q^{\prime}\right)$, respectively. By commensurability their intersection has finite index, so there exist nonzero integers $k, l$ such that

$$
A^{k}=A^{\prime l} \text {. }
$$

Proposition 3.6 implies that $a^{\prime}$ is isotopic with $a$ or with $a^{-1}$. Since $N$ and $N^{\prime}$ are regular neighborhoods, respectively, of $a$ and $a^{\prime}$, it follows that $N$ and $N^{\prime}$ are isotopic in this case.

Case 2: $\left(N^{\prime}, Q^{\prime}\right)$ is $\left(D^{2}, 2\right)$. Choose notation as in Case 1 , except that $a^{\prime}$ now denotes the boundary curve of $N^{\prime}$. Again we have nonzero $k$ and $l$ so that $A^{k}=A^{\prime l}$, and we conclude that $a^{\prime}$ is isotopic with $a^{ \pm 1}$. After an isotopy, we may assume $a^{\prime}$ equals a boundary component of $N$. It then follows that $N^{\prime}$ is a component of the exterior of $N$.

Definition: A doubled pantalon is the marked surface obtained by pasting together two pantalons of the same type along their boundaries (see Figure 19). More specifically, if $(M, P)$ is a marked surface and $N$ a subsurface of $M$, the triple $(M, N, P)$ is called a doubled pantalon in each of the cases:

Type I: $M \cong S^{2}, \quad N \cong D^{2}, \quad|P|=4, \quad|P \cap N|=2$;

Type II: $M \cong S^{1} \times S^{1} \supset S^{1} \times I \cong N, \quad|P|=2, \quad|P \cap N|=1$;

Type III: $M=$ closed surface of genus two, $P=$ empty set, $N \cong \overline{M \backslash N}=$ type III pantalons.

One reason to be interested in doubled pantalons is that they provide examples of nonisotopic subsurfaces inducing commensurable geometric subgroups, which we will see to be an exceptional case.

Proposition 6.4 Suppose that $(M, N, P)$ is a doubled pantalon, and let $N^{\prime}=$ $\overline{M \backslash N}$. Then $\mathcal{M}(N, N \cap P)$ and $\mathcal{M}\left(N^{\prime}, N^{\prime} \cap P\right)$ inject in $\mathcal{M}(M, P)$, and are commensurable subgroups. The subgroups are equal in the case of Types II or III. For Type I, the intersection $\mathcal{M}(N, N \cap P) \cap \mathcal{M}\left(N^{\prime}, N^{\prime} \cap P\right)$ has index two in each of $\mathcal{M}(N, N \cap P)$ and $\mathcal{M}\left(N^{\prime}, N^{\prime} \cap P\right)$. In each of the three types, the subsurfaces $N$ and $N^{\prime}$ are non-isotopic in $M$ (rel $\left.P\right)$. 


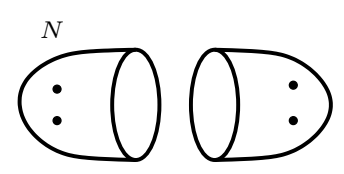

Type I

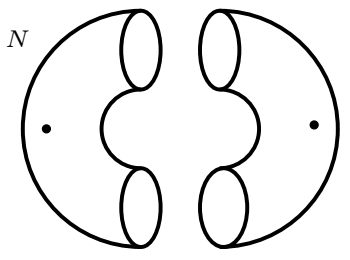

Type II

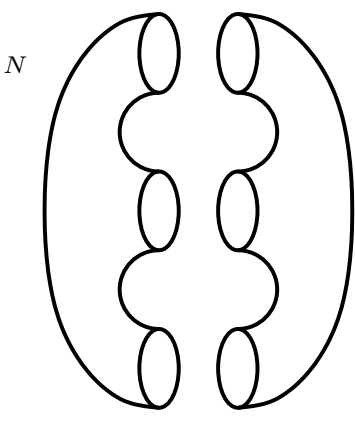

Type III

Figure 19: Doubled pantalons.

Proof: In the case of doubled pantalons of Type II or III, the two geometric subgroups both equal the free abelian group generated by twists along the common boundary of $N$ and $N^{\prime}$. This has rank 2 or 3 , respectively. For Type I, the generator of $\mathcal{M}(N, N \cap P)$ is a half-twist interchanging the two points $N \cap P$, which clearly does not belong to $\mathcal{M}\left(N^{\prime}, N^{\prime} \cap P\right)$, all of whose elements fix $N \cap P$ pointwise. Likewise the generator of $\mathcal{M}\left(N^{\prime}, N^{\prime} \cap P\right)$ is not in $\mathcal{M}(N, N \cap P)$. But the squares of the generators, being a Dehn twist along the common boundary, coincide. The question of isotopy is clear for types $I$ and $I I$, because the surfaces $N$ and $N^{\prime}$ enclose different marked points. In type III, the two pantalons, $N$ and $N^{\prime}$ are nonisotopic, too. For an isotopy taking $N$ to $N^{\prime}$ would take the boundary to itself, but with orientation reversed. But a simple homological calculation shows that if $a, b, c$ are the (oriented) boundary curves of $N, a$ cannot be isotopic with $a^{-1}, b^{-1}$ or $c^{-1}$.

In the following, consider two connected marked injective subsurfaces $(N, Q)$, $\left(N^{\prime}, Q^{\prime}\right) \subset(M, P)$, it being understood that $Q=N \cap P$ and $Q^{\prime}=N^{\prime} \cap P$. We will refer to the following four statements:

a) $\mathcal{M}(N, Q)$ and $\mathcal{M}\left(N^{\prime}, Q^{\prime}\right)$ are commensurable subgroups of $\mathcal{M}(M, P)$;

b) $\mathcal{M}(N, Q)=\mathcal{M}\left(N^{\prime}, Q^{\prime}\right)$;

c) $(N, Q)$ and $\left(N^{\prime}, Q^{\prime}\right)$ are isotopic;

d) $(N, Q)$ is isotopic with either $\left(N^{\prime}, Q^{\prime}\right)$ or $\left(\overline{M \backslash N^{\prime}}, P \backslash Q^{\prime}\right)$.

Theorem 6.5 Suppose that $N$ and $N^{\prime}$ are injective subsurfaces of $M$ and that $(N, Q) \neq\left(S^{1} \times I, 0\right) \neq\left(N^{\prime}, Q^{\prime}\right)$. Then:

i) if $(M, N, P)$ is not a doubled pantalon, $(a) \Leftrightarrow(b) \Leftrightarrow(c)$;

ii) if $(M, N, P)$ is a doubled pantalon of Type II or III, $(a) \Leftrightarrow(b) \Leftrightarrow(d)$;

iii) if $(M, N, P)$ is a doubled pantalon of Type $I,(b) \Leftrightarrow(c) \Rightarrow(a) \Leftrightarrow(d)$. 
Proof: It is obvious that $(c) \Rightarrow(b) \Rightarrow(a)$ in all three cases. Before breaking into cases, let $d_{1}, \ldots, d_{l}: S^{1} \rightarrow M \backslash P$ denote the boundary components of $N$ and similarly $d_{1}^{\prime}, \ldots, d_{l^{\prime}}^{\prime}$ the components of $\partial N^{\prime}$. Let $\left\{a_{i}\right\}$ be curves in $N$ defining a pantalon decomposition of $N$ (see Figure 20). Let $N_{j}$ denote the components of $\overline{M \backslash N}$. Choose curves $b_{j k}$ giving a pantalon decomposition of $\left(N_{j}, N_{j} \cap P\right)$ for $\left(N_{j}, N_{j} \cap P\right) \neq\left(S^{1} \times I, \emptyset\right)$.

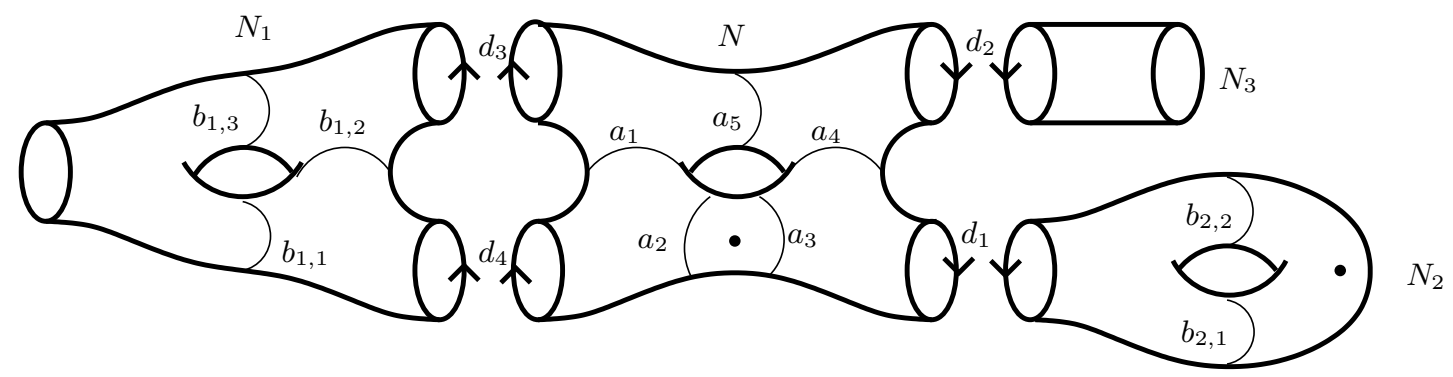

Figure 20: Pantalon decomposition of $N$ and its complement.

We wish to show that in all cases $(a) \Rightarrow(d)$. So we assume commensurability of the geometric subgroups $\mathcal{M}(N, Q)$ and $\mathcal{M}\left(N^{\prime}, Q^{\prime}\right)$; the strategy is to show that after an isotopy, the boundaries of $N$ and $N^{\prime}$ can be made to coincide. We break the argument into two steps.

Step 1: Each component $d_{j}^{\prime}$ of $\partial N^{\prime}$ is isotopic (in $M$, rel $P$ ) with a component $\left(d_{i}\right)^{ \pm 1}$ of $\partial N$, and vice-versa.

Proof of Step 1: First note that we may assume in this step that $\partial M=\emptyset$, by the trick used before: adjoin a genus one surface to each boundary component of $M$ to obtain $\hat{M} \cdot \mathcal{M}(M, P)$ injects in $\mathcal{M}(\hat{M}, P)$, so the subgroups $\mathcal{M}(N, Q)$ and $\mathcal{M}\left(N^{\prime}, Q^{\prime}\right)$ are unchanged, and the desired conclusion of step 1 , if true in $\hat{M}$, will also hold in $M$ by Proposition 3.5. In particular, since $N$ is injective, none of the $N_{j}$ is a cylinder with $N_{j} \cap P=\emptyset$, and the union of all the $a_{i}, b_{j k}$ and $d_{i}$ gives a pantalon decomposition of $M$. Let $A_{i}, B_{j k}$, and $D_{i}$ denote the Dehn twists about the respective curves $a_{i}, b_{j k}, d_{i}$. Let $d^{\prime}=d_{j}^{\prime}$ be any boundary component of $N^{\prime}$, and $D^{\prime}$ the element of $\mathcal{M}\left(N^{\prime}, Q^{\prime}\right) \subset \mathcal{M}(M, P)$ represented by a Dehn twist along $d^{\prime}$. We now calculate some intersection numbers:

$I\left(a_{i}, d^{\prime}\right)=0$. Reason: Being a twist on a boundary curve, $D^{\prime}$ is central in $\mathcal{M}\left(N^{\prime}, Q^{\prime}\right)$, though perhaps not in $\mathcal{M}(M, P)$. By commensurability and Proposition 6.1 since $A_{i} \in \mathcal{M}(N, Q)$ there is an integer $s \neq 0$ such that $A_{i}^{s} \in \mathcal{M}\left(N^{\prime}, Q^{\prime}\right)$. It follows that $A_{i}^{s}$ and $D^{\prime}$ commute. Then Proposition 3.7 implies that $I\left(a_{i}, d^{\prime}\right)=0$.

$I\left(b_{j k}, d^{\prime}\right)=0$. This is shown similarly, noting that $B_{j k}$ commutes with all of $\mathcal{M}(N, Q)$, and by commensurability, a nonzero power of $D^{\prime}$ is in $\mathcal{M}(N, Q)$. 
$I\left(d_{i}, d^{\prime}\right)=0$ for all $i$. As in the previous case, a power of $D^{\prime}$ belongs to $\mathcal{M}(N, Q)$ and so commutes with $D_{i}$, a twist of $\partial N$.

Now Proposition 6.2 implies that $d^{\prime}$ may be assumed, after an isotopy, to be disjoint from the $a_{i}, b_{j k}, d_{i}$, i. e. $d^{\prime}$ lies entirely inside one of the pantalons in the decomposition of $M$ corresponding to those curves. Being essential, $d^{\prime}$ is isotopic with one of the boundary curves of that pantalon (or its inverse). We have concluded that, up to isotopy of $M$ rel $P$, we have one (and only one) of the following possibilities, for some $i, j, k$ :

$$
d^{\prime} \simeq a_{i}^{ \pm 1}, \quad d^{\prime} \simeq b_{j k}^{ \pm 1}, \text { or } \quad d^{\prime} \simeq d_{i}^{ \pm 1} .
$$

First assume $d^{\prime} \simeq a_{i}^{ \pm 1}$. Since $a_{i}$ is not isotopic with a boundary component of $N$, there exists a generic simple closed curve $e$ in $N \backslash Q$ such that $I\left(d^{\prime}, e\right)=I\left(a_{i}, e\right)>0$. The Dehn twist $E$ along $e$ is an element of $\mathcal{M}(N, Q)$, a subgroup commensurable with $\mathcal{M}\left(N^{\prime}, Q^{\prime}\right)$, so there exists $t>0$ such that $E^{t} \in \mathcal{M}\left(N^{\prime}, Q^{\prime}\right)$. Recalling that $D^{\prime}$ is central in $\mathcal{M}\left(N^{\prime}, Q^{\prime}\right), D^{\prime}$ and $E^{t}$ commute. By Proposition 3.7, $I\left(d^{\prime}, e\right)=0$, a contradiction.

Next assume $d^{\prime} \simeq b_{j k}^{ \pm 1}$, one of the pantalon curves of the component $N_{j}$ of the complement of $N$. As above, there is a simple closed curve $e$ in $N_{j} \backslash\left(N_{j} \cap P\right)$ with $I\left(d^{\prime}, e\right)=I\left(b_{j k}, e\right)>0$. Noting that the Dehn twist $E$ commutes with all elements of $\mathcal{M}(N, N \cap P)$ and that a power of $D^{\prime}$ belongs to $\mathcal{M}(N, N \cap P)$, we obtain again the contradiction that $I\left(d^{\prime}, e\right)=0$. Therefore $d^{\prime} \simeq d_{i}^{ \pm 1}$. So Step 1 is established, noting that by symmetry, any $d_{i}$ is isotopic to some $d_{k}^{\prime}$.

Step 2: Now, the boundary of $M$ is not assumed to be necessarily empty. If, as in the hypothesis, $(N, Q) \neq\left(S^{1} \times I, 0\right) \neq\left(N^{\prime}, Q^{\prime}\right)$, the curves $d_{1}, \ldots, d_{l}$ are isotopically distinct, and likewise $d_{1}^{\prime}, \ldots, d_{l^{\prime}}^{\prime}$. We conclude that $l=l^{\prime}$ and, after renumbering, each $d_{i}^{\prime}$ is isotopic in $M$ rel $P$, with $d_{i}$ or $d_{i}^{-1}$. By Proposition 3.10 there is an isotopy of $M \backslash P$ taking $\partial N$ to $\partial N^{\prime}$. It follows that $(a) \Rightarrow(d)$ in all cases.

We now show that the possibility $N=\overline{M \backslash N^{\prime}}$ can occur only if $(N, Q)$ is a pantalon in which case $(M, N, P)$ is a doubled pantalon. Suppose that $(N, Q)$ is not a pantalon (or $\left(S^{1} \times I, 0\right),\left(D^{2}, 0\right),\left(D^{2}, 1\right)$ which are excluded by assumption). Then there exist curves $a, b: S^{1} \rightarrow N \backslash Q$ with $I(a, b)>0$. Let $A, B \in \mathcal{M}(N, Q)$ denote the corresponding Dehn twists. By commensurability, $A^{k} \in \mathcal{M}\left(N^{\prime}, Q^{\prime}\right)$ for some $k>0$. But by disjointness, all elements of $\mathcal{M}\left(N^{\prime}, Q^{\prime}\right)$ commute with all elements of $\mathcal{M}(N, Q)$, so $A^{k}$ and $B$ commute. Proposition 3.7 implies $I(a, b)=0$, a contradiction.

In summary, we have shown that in case (i), $(c) \Rightarrow(b) \Rightarrow(a) \Rightarrow(c)$. Cases (ii) and (iii) also follow from the above arguments and Proposition 6.4. 


\section{Normalizers and Commensurators}

The commensurator of a subgroup $H$ of a group $G$ is

$$
\operatorname{Com}_{G}(H)=\left\{g \in G: g^{-1} H g \text { and } H \text { are commensurable }\right\} \text {, }
$$

the normalizer of $H$ in $G$ is

$$
\mathcal{N}_{G}(H)=\left\{g \in G: g^{-1} H g=H\right\},
$$

and the centralizer of $H$ in $G$ is

$$
\mathcal{Z}_{G}(H)=\{g \in G: g h=h g \text { for all } h \in H\} .
$$

In general we have

$$
\mathcal{Z}_{G}(H) \subset \mathcal{N}_{G}(H) \subset \operatorname{Com}_{G}(H) . \quad \text { Also } H \subset \mathcal{N}_{G}(H) .
$$

For any subsurface $N$ of $M$, we define the stabilizer $\operatorname{Stab}(N)$ as

$$
\operatorname{Stab}(N)=\{[h] \in \mathcal{M}(M, P): h(N) \text { is isotopic to } N \text { in } M \text { rel } P \cup \partial M\} .
$$

Noting that $h \simeq h^{\prime}$ implies $h(N) \simeq h^{\prime}(N)$, we see this is a well-defined subgroup of $\mathcal{M}(M, P)$.

As in the previous section, the doubled pantalons are exceptional cases, so we make the following constructions. Let $(M, N, P)$ be a doubled pantalon of type I, II or III, embedded in the $x y z$-space symmetrically with respect to 180 degree rotation $s: M \rightarrow M$ about the $x$-axis, and so that $(N, N \cap P)$ is the intersection of $(M, P)$ with the half-space $y \geq 0$ (see Figure 21). The map $s$ interchanges $N$ and $\overline{M \backslash N}$, and reverses the orientation of $\partial N$. We will call $\sigma=[s]: \mathcal{M}(M, P) \rightarrow \mathcal{M}(M, P)$, the exchange map, for each of the three types of doubled pantalon.

Theorem 7.1 Let $N \subset M$ be an injective subsurface and denote $G=\mathcal{M}(M, P)$ and $H=\mathcal{M}(N, N \cap P)$.

i) If $(M, N, P)$ is not a doubled pantalon, then

$$
\operatorname{Com}_{G}(H)=\mathcal{N}_{G}(H)=\operatorname{Stab}(N) .
$$

ii) If $(M, N, P)$ is a doubled pantalon of type II or III, then

$$
\operatorname{Com}_{G}(H)=\mathcal{N}_{G}(H)=\operatorname{Stab}(N) \times\langle\sigma\rangle .
$$

iii) If $(M, N, P)$ is a doubled pantalon of type $I$, then

$$
\operatorname{Com}_{G}(H)=\operatorname{Stab}(N) \rtimes\langle\sigma\rangle \text { and } \mathcal{N}_{G}(H)=\operatorname{Stab}(N) .
$$

Here $\langle\sigma\rangle$ denotes the cyclic subgroup of order 2 generated by the exchange $\sigma$ of $(M, N, P)$ and $\operatorname{Stab}(N)$ is normal in the semidirect product. 


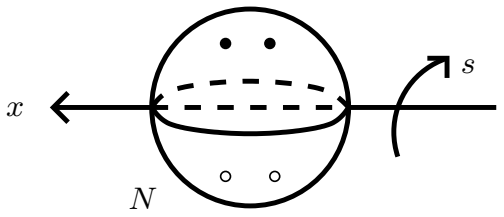

Type I

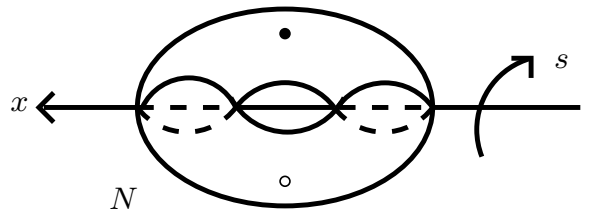

Type II

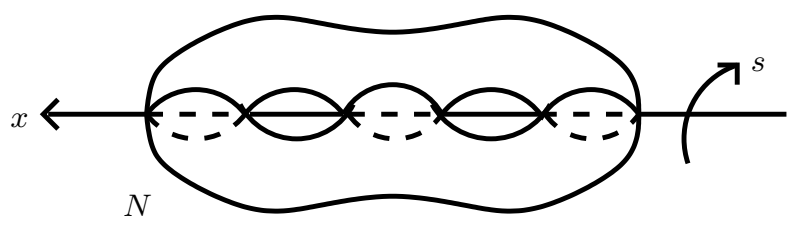

Type III

Figure 21: Exchange map for doubled pantalons.

Proof: Let $\xi \in \operatorname{Com}_{G}(H)$ and let $h \in \mathcal{H}(M, P)$ represent $\xi$. Note that

$$
\xi \mathcal{M}(N, P \cap N) \xi^{-1}=\mathcal{M}(h(N), P \cap h(N)) .
$$

First suppose $(M, N, P)$ is not a doubled pantalon, and if $N$ is a cylinder assume $P \cap N$ is nonempty. Since the groups $\mathcal{M}(N, P \cap N)$ and $\mathcal{M}(h(N), P \cap h(N))$ are commensurable, Theorem 6.5 implies that $\mathcal{M}(N, P \cap N)=\mathcal{M}(h(N), P \cap h(N))$ and that $h(N)$ is isotopic to $N$ rel $P$. This shows that $\operatorname{Com}_{G}(H)=\mathcal{N}_{G}(H)=\operatorname{Stab}(N)$.

Next suppose that $N$ is a cylinder and $P \cap N$ is empty. Then $h(N)$ is also a cylinder and by Proposition $6.3 \mathcal{M}(N, P \cap N)=\mathcal{M}(h(N), P \cap h(N))$ and $h(N)$ is isotopic to $N$ rel $P$. Again we conclude $\operatorname{Com}_{G}(H)=\mathcal{N}_{G}(H)=\operatorname{Stab}(N)$.

Now assume that $(M, N, P)$ is a doubled pantalon of type II or III. By Theorem 6.5, $\mathcal{M}(N, P \cap N)=\mathcal{M}(h(N), P \cap h(N))$ and $h(N)$ is isotopic to $N$ or $\overline{M \backslash N}$ rel $P$. Therefore $\operatorname{Com}_{G}(H)=\mathcal{N}_{G}(H)$, but this group is bigger than $\operatorname{Stab}(N)$; for example $\sigma$ belongs to the normalizer, but does not stabilize $N$.

We can define an epimorphism $f: \operatorname{Com}_{G}(H) \rightarrow \mathbf{Z} / 2 \mathbf{Z}$ by $f([h])=0$ if $h(N)$ is isotopic to $N$ and $f([h])=1$ if $h(N)$ is isotopic to $\overline{M \backslash N}$. The kernel of $f$ is $\operatorname{Stab}(N)$. The homomorphism $f$ has a section $s: \mathbf{Z} / 2 \mathbf{Z} \rightarrow \operatorname{Com}_{G}(H)$ given by $s(0)=[i d], s(1)=\sigma$, where $\sigma$ is the exchange of $(M, N, P)$. It follows that $\operatorname{Com}_{G}(H)=\operatorname{Stab}(N) \rtimes\langle\sigma\rangle$. By Propositions 5.3 and 5.5, $\sigma$ is in the centre of $\mathcal{M}(M, P)$, so the product is, in fact, a direct product.

Finally, consider the case that $(M, N, P)$ is a doubled pantalon of type I. By Theorem 6.5, $h(N)$ is isotopic with $N$ or $\overline{M \backslash N}$. Moreover, if $\xi \in \mathcal{N}_{G}(H)$, then 
$h(N)$ is isotopic with $N$, so we conclude that $\mathcal{N}_{G}(H)=\operatorname{Stab}(N)$. By defining the homomorphism $f$ and its section $s$ exactly as above, we also conclude that $\operatorname{Com}_{G}(H)=\operatorname{Stab}(N) \rtimes\langle\sigma\rangle$.

We proceed now to study the group $\operatorname{Stab}(N)$ which, as seen before, determines the commensurator and the normalizer of $\mathcal{M}(N, N \cap P)$ in $\mathcal{M}(M, P)$.

We first state the following proposition which can be proved in the same manner as Theorem 4.1.

Proposition 7.2 Let $(N, Q)$ and $\left(N^{\prime}, Q^{\prime}\right)$ be marked surfaces. Choose l boundary components $c_{1}, \ldots, c_{l}$ of $N$ and $l$ boundary components $c_{1}^{\prime}, \ldots, c_{l}^{\prime}$ of $N^{\prime}$, and denote by $C_{i}$ and $C_{i}^{\prime}$ the Dehn twists corresponding to $c_{i}$ and $c_{i}^{\prime}$ in $\mathcal{M}(N, Q)$ and $\mathcal{M}\left(N^{\prime}, Q^{\prime}\right)$, respectively. Let $M$ be the surface obtained by pasting together $N$ and $N^{\prime}$ along the curves $c_{i}$ and $c_{i}^{\prime-1}$ for all $i=1, \ldots, l$, and let $P=Q \cup Q^{\prime} \subset M$ (see Figure 29). Assume that $|Q| \geq 2$ if $N$ is a disk and $|Q| \geq 1$ if $N$ is an annulus. Assume the same for $N^{\prime}$ and $Q^{\prime}$. Consider the homomorphism $\phi: \mathcal{M}(N, Q) \times \mathcal{M}\left(N^{\prime}, Q^{\prime}\right) \rightarrow \mathcal{M}(M, P)$ induced by the inclusions of $N$ and $N^{\prime}$ in $M$. Then the kernel of $\phi$ is generated by

$$
\left\{\left(C_{1}, C_{1}^{\prime-1}\right), \ldots,\left(C_{l}, C_{l}^{\prime-1}\right)\right\}
$$

and is isomorphic with $\mathbf{Z}^{l}$.

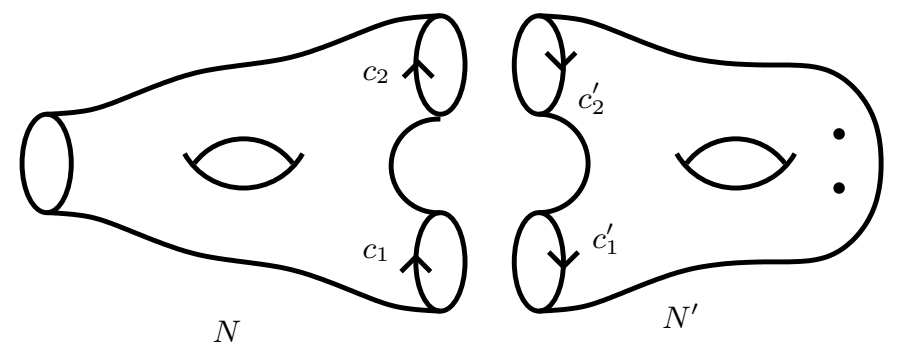

Figure 22: Marked surfaces whose union is $M$.

Let $(M, P)$ be a marked surface, and let $N$ be an injective subsurface of $M$. Let $c_{1}, \ldots, c_{l}$ denote the boundary components of $N$ and $\Sigma_{l}$ the symmetric group of $\{1, \ldots, l\}$. There is a natural homomorphism $\tau: \operatorname{Stab}(N) \rightarrow \Sigma_{l}$ which associates to $[h] \in \operatorname{Stab}(N)$ the unique $\sigma \in \Sigma_{l}$ such that $h\left(c_{i}\right)$ is isotopic to $c_{\sigma(i)}$. Here we assume that the curves $c_{i}$ are provided with the orientation induced by the one of $N$. So, since $h(N)$ is isotopic to $N$, such a $\sigma$ exists and is unique, even if $N$ 
is an annulus and $N \cap P=\emptyset$. The homomorphism $\tau$ is not surjective in general. However, one can explicitly describe its image which depends on the topology of the complement of $N \backslash N \cap P$ in $M \backslash P$. This image is long and tedious to describe and we let the reader off this description.

Let $N_{1}, \ldots, N_{r}$ denote the connected components of $\overline{M \backslash N}$. The inclusions of the $N_{i}$ in $M$ and of $N$ in $M$ induce a homomorphism

$$
\phi: \mathcal{M}(N, N \cap P) \times \mathcal{M}\left(N_{1}, N_{1} \cap P\right) \times \ldots \times \mathcal{M}\left(N_{r}, N_{r} \cap P\right) \rightarrow \mathcal{M}(M, P) .
$$

Assume that $N$ is not an annulus with $N \cap P=\emptyset$. Let $C_{i}$ denote the Dehn twist in $\mathcal{M}(N, N \cap P)$ corresponding to $c_{i}, N_{j}$ the component of $\overline{M \backslash N}$ having $c_{i}$ as boundary component, and $C_{i}^{\prime}$ the Dehn twist in $\mathcal{M}\left(N_{j}, N_{j} \cap P\right)$ corresponding to $c_{i}$. Then, by Proposition 7.2, the kernel of $\phi$ is generated by

$$
\left\{C_{1} C_{1}^{\prime-1}, \ldots, C_{l} C_{l}^{\prime-1}\right\}
$$

and is a copy of $\mathbf{Z}^{l}$. The image of $\phi$ is obviously included in the kernel of $\tau$ and, by Proposition 3.10, any element of the kernel of $\tau$ belongs in the image of $\phi$. So, we have proved:

Theorem 7.3 Let $(N, N \cap P)$ be a marked injective subsurface of $(M, P)$ different from $\left(S^{1} \times I, \emptyset\right)$, and let $\tau$ and $\phi$ be the homomorphisms given above. Then we have the exact sequence

$1 \rightarrow \mathbf{Z}^{l} \rightarrow \mathcal{M}(N, N \cap P) \times \mathcal{M}\left(N_{1}, N_{1} \cap P\right) \times \ldots \times \mathcal{M}\left(N_{r}, N_{r} \cap P\right) \stackrel{\phi}{\rightarrow} \operatorname{Stab}(N) \stackrel{\tau}{\rightarrow} \Sigma_{l}$.

Proposition 7.4 Assume that $N$ is an annulus, $N \cap P=\emptyset$, and there is no boundary component of $N$ which is the boundary of a disk intersecting $P$ in less than two points.

i) Suppose $M$ is a torus and $P$ is empty. Then

$$
\operatorname{Stab}(N)=\mathcal{M}(N) \times\langle\sigma\rangle,
$$

where $\sigma$ is the element in $\mathcal{M}(M)$ of order two which generates the centre.

ii) Suppose $\overline{M \backslash N}$ has a unique connected component, $N_{1}$, and $(M, P) \neq$ $\left(T^{2}, \emptyset\right)$ (see Figure 23. i). Then we have an exact sequence

$$
1 \rightarrow \mathbf{Z} \rightarrow \mathcal{M}\left(N_{1}, P\right) \rightarrow \operatorname{Stab}(N) \stackrel{\tau}{\rightarrow} \Sigma_{2} \rightarrow 1 .
$$

iii) Suppose $\overline{M \backslash N}$ has two connected components, $N_{1}$ and $N_{2}$ (see Figure 23. ii). Then we have an exact sequence

$$
1 \rightarrow \mathbf{Z} \rightarrow \mathcal{M}\left(N_{1}, N_{1} \cap P\right) \times \mathcal{M}\left(N_{2}, N_{2} \cap P\right) \rightarrow \operatorname{Stab}(N) \stackrel{\tau}{\rightarrow} \Sigma_{2} .
$$



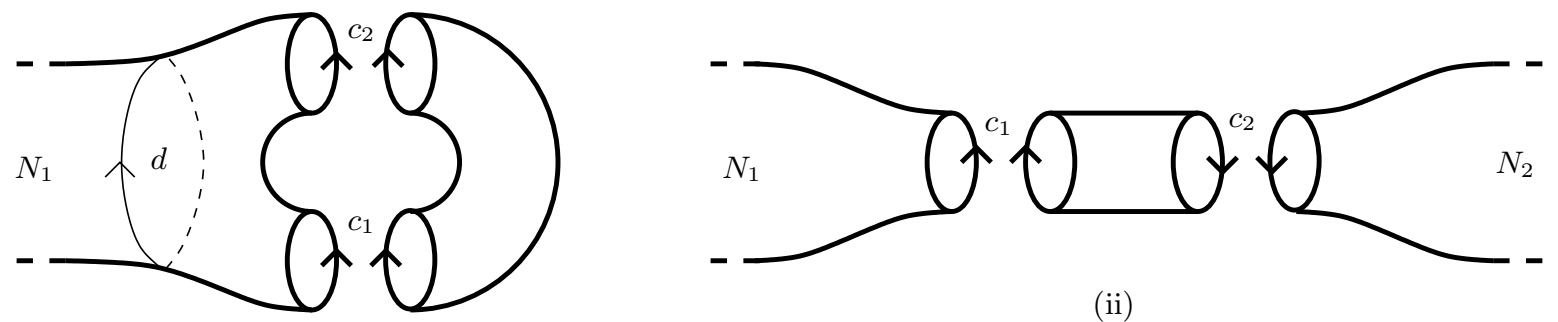

(ii)

(i)

Figure 23: The annulus $N$ of Proposition 7.4.

Proof: First, suppose that $M$ is a torus and $P$ is empty. By the structure of the mapping class group of an annulus, the kernel of $\tau$ is $\mathcal{M}(N) \simeq \mathbf{Z}$. Moreover, $\sigma \in \operatorname{Stab}(N)$ and $\sigma$ permutes (up to isotopy) the boundary components of $N$, thus $\tau$ is surjective and the map $(1,2) \mapsto \sigma$ gives a section of $\tau$. Since $\sigma$ is central in $\mathcal{M}(M)$, it follows that $\operatorname{Stab}(N)=\mathcal{M}(N) \times\langle\sigma\rangle$.

Assume now that $\overline{M \backslash N}$ has a unique connected component, $N_{1}$, and $(M, P) \neq$ $\left(T^{2}, \emptyset\right)$. Let $c_{1}, c_{2}$ denote the boundary components of $N$. We can cut $N_{1}$ along some closed essential curve $d$ into two subsurfaces such that one of them is a pantalon of type III having $d, c_{1}$ and $c_{2}$ as boundary components (see Figure 23.i). Pasting this pantalon with $N$ one obtains a genus one surface with one boundary component, $d$. Let $\rho$ be the half-twist of this surface of genus one along $d$ relative to $c_{1}$, as defined in Section 5. Then $\rho \in \operatorname{Stab}(N)$ and $\tau(\rho)=(1,2)$. This shows that $\tau$ is surjective. Let $C_{i}$ denote the Dehn twist along $c_{i}$ in $\mathcal{M}\left(N_{1}, P\right)$, $\iota: \mathcal{M}\left(N_{1}, P\right) \rightarrow \operatorname{Stab}(N)$ the homomorphism induced by the inclusion of $N_{1}$ in $M$, and $\theta: \mathbf{Z} \rightarrow \mathcal{M}\left(N_{1}, P\right)$ the homomorphism defined by $\theta(1)=C_{1} C_{2}^{-1}$. Then the equality $\operatorname{Im} \iota=\operatorname{Ker} \tau$ follows from Proposition 3.10, and Theorem 4.1 implies $\operatorname{Ker} \theta=\{0\}$ and $\operatorname{Im} \theta=\operatorname{Ker} \iota$.

Assume now that $\overline{M \backslash N}$ has two connected components, $N_{1}$ and $N_{2}$. Let $c_{i}$ denote the common boundary component of $N$ and $N_{i}, C_{i}$ the Dehn twist along $c_{i}$ in $\mathcal{M}\left(N_{i}, N_{i} \cap P\right)$,

$$
\iota: \mathcal{M}\left(N_{1}, N_{1} \cap P\right) \times \mathcal{M}\left(N_{2}, N_{2} \cap P\right) \rightarrow \operatorname{Stab}(N)
$$

the homomorphism induced by the inclusions of $N_{1}$ and $N_{2}$ in $M$, and

$$
\theta: \mathbf{Z} \rightarrow \mathcal{M}\left(N_{1}, N_{1} \cap P\right) \times \mathcal{M}\left(N_{2}, N_{2} \cap P\right)
$$

the homomorphism defined by $\theta(1)=\left(C_{1}, C_{2}^{-1}\right)$. The equality $\operatorname{Im} \iota=\operatorname{Ker} \tau$ and the inclusion $\operatorname{Im} \theta \subset$ Ker $\iota$ are obvious, and the injectivity of $\theta$ follows from Corollary 
3.9. So, it remains to prove the inclusion Ker $\iota \subset \operatorname{Im} \theta$. If $\left(N_{1}, N_{1} \cap P\right)=\left(S^{1} \times I, \emptyset\right)$, then $\mathcal{M}\left(N_{1}, N_{1} \cap P\right)=\mathcal{M}(N)$ is the infinite cyclic subgroup generated by $C_{1}=C_{2}$, and therefore, since $\mathcal{M}\left(N_{2}, N_{2} \cap P\right)$ injects in $\mathcal{M}(M, P)$ by Theorem 4.1, Ker $\iota \subset$ $\operatorname{Im} \theta$. If $\left(N_{1}, N_{1} \cap P\right) \neq\left(S^{1} \times I, \emptyset\right) \neq\left(N_{2}, N_{2} \cap P\right)$, then the inclusion Ker $\iota \subset \operatorname{Im} \theta$ follows from Proposition 7.2.

\section{Centralizers}

Theorem 8.1 Let $N \subset M$ be an injective subsurface and denote $G=\mathcal{M}(M, P)$ and $H=\mathcal{M}(N, N \cap P)$.

i) If $N$ is an annulus and $N \cap P$ is empty, then

$$
\operatorname{Com}_{G}(H)=\mathcal{N}_{G}(H)=\mathcal{Z}_{G}(H)=\operatorname{Stab}(N) .
$$

ii) If $(M, N, P)$ is a doubled pantalon of type II or III, then

$$
\mathcal{Z}_{G}(H)=\mathcal{M}(N, N \cap P) \times\langle\sigma\rangle,
$$

where $\langle\sigma\rangle$ is the cyclic subgroup of order two generated by the exchange $\sigma$ of $(M, N, P)$.

iii) Suppose that $(N, N \cap P)$ is not as in (i) or (ii). Let $N_{1}, \ldots, N_{r}$ denote the connected components of $\overline{M \backslash N}$. Then we have the exact sequence

$1 \rightarrow \mathbf{Z}^{l} \rightarrow \mathcal{Z} \mathcal{M}(N, N \cap P) \times \mathcal{M}\left(N_{1}, N_{1} \cap P\right) \times \ldots \times \mathcal{M}\left(N_{r}, N_{r} \cap P\right) \stackrel{\phi}{\rightarrow} \mathcal{Z}_{G}(H) \rightarrow 1$,

where $l$ is the number of components of $\partial N$ and $\phi$ is the homomorphism defined in Section 7. Moreover, assuming that $|N \cap P| \geq 3$ if $N$ is a disk and $|N \cap P| \geq 1$ if $N$ is a genus one surface with one boundary component, the restriction

$$
\phi: \mathcal{M}\left(N_{1}, N_{1} \cap P\right) \times \ldots \times \mathcal{M}\left(N_{r}, N_{r} \cap P\right) \rightarrow \mathcal{Z}_{G}(H)
$$

of $\phi$ to $\mathcal{M}\left(N_{1}, N_{1} \cap P\right) \times \ldots \times \mathcal{M}\left(N_{r}, N_{r} \cap P\right)$ is an isomorphism.

Proof: Suppose that $N$ is an annulus and $N \cap P$ is empty. The equalities $\operatorname{Com}_{G}(H)=\mathcal{N}_{G}(H)=\operatorname{Stab}(N)$ are proved in Theorem 7.1 and the inclusion $\mathcal{Z}_{G}(H) \subset \mathcal{N}_{G}(H)$ is obvious. So, it remains to prove $\operatorname{Stab}(N) \subset \mathcal{Z}_{G}(H)$. Let $c_{1}, c_{2}$ denote the boundary curves of $N$. The Dehn twists $C_{1}$ and $C_{2}$ along $c_{1}$ and $c_{2}$, respectively, coincide and generate $\mathcal{M}(N)$. Let $\xi \in \operatorname{Stab}(N)$ and let $h \in \mathcal{H}(M, P)$ represent $\xi$. Then $h \circ c_{1}$ is isotopic with $c_{1}$ or $c_{2}$, thus $\xi C_{1} \xi^{-1}=C_{1}$ or $C_{2}=C_{1}$, therefore $\xi \in \mathcal{Z}_{G}(H)$. 
Suppose now that $(N, N \cap P)$ is not as in (i) or (ii). We know by Theorem 7.1 that $\mathcal{Z}_{G}(H) \subset \mathcal{N}_{G}(H)=\operatorname{Stab}(N)$. Consider the exact sequence of Theorem 7.3. Let $c_{1}, \ldots, c_{l}$ denote the boundary components of $N$ and $C_{i}$ the Dehn twist in $\mathcal{M}(M, P)$ corresponding to $c_{i}$. Let $\xi \in \mathcal{Z}_{G}(H)$ and let $h \in \mathcal{H}(M, P)$ represent $\xi$. The transformation $h$ cannot permute the $c_{i}$ because $\xi C_{i} \xi^{-1}$ is the Dehn twist along $h \circ c_{i}$, the equality $\xi C_{i} \xi^{-1}=C_{i}$ implies by Proposition 3.6 that $h \circ c_{i}$ is isotopic with $c_{i}$ or $c_{i}^{-1}$, and $(N, N \cap P) \neq\left(S^{1} \times I, \emptyset\right)$ together with the injectivity of $N$ imply that $c_{i}^{-1}$ is not isotopic with some $c_{j}, j \neq i$. So, $\mathcal{Z}_{G}(H) \subset \operatorname{Ker} \tau=\operatorname{Im} \phi$. Then the exact sequence

$1 \rightarrow \mathbf{Z}^{l} \rightarrow \mathcal{Z} \mathcal{M}(N, N \cap P) \times \mathcal{M}\left(N_{1}, N_{1} \cap P\right) \times \ldots \times \mathcal{M}\left(N_{r}, N_{r} \cap P\right) \stackrel{\phi}{\rightarrow} \mathcal{Z}_{G}(H) \rightarrow 1$

is a straightforward consequence of the exact sequence of Theorem 7.3.

Suppose now in addition that $|N \cap P| \geq 3$ if $N$ is a disk and $|N \cap P| \geq 1$ if $N$ is a genus one surface with one boundary component. Then, by Theorem 5.6, the centre of $\mathcal{M}(N, N \cap P)$ is the free abelian group of rank $l$ generated by $\left\{C_{1}, \ldots, C_{l}\right\}$. Thus it follows from the expression of the kernel of $\phi$ given in the proof of Theorem 7.3 that the restriction of $\phi$ to $\mathcal{M}\left(N_{1}, N_{1} \cap P\right) \times \ldots \times \mathcal{M}\left(N_{r}, N_{r} \cap P\right)$ is an isomorphism.

Assume now that $(M, N, P)$ is a doubled pantalon of type II or III. Let $\xi \in$ $\mathcal{Z}_{G}(H)$ and let $h \in \mathcal{H}(M, P)$ represent $\xi$. We show as above that, if $h$ stabilizes $N$ (up to isotopy), then $h$ fixes (up to isotopy) the boundary components of $N$, and therefore $h \in \mathcal{M}(N, N \cap P)=\mathcal{M}(\overline{M \backslash N}, \overline{M \backslash N} \cap P)$. So,

$$
\mathcal{Z}_{G}(H) \cap \operatorname{Stab}(N)=\mathcal{M}(N, N \cap P) .
$$

The exchange $\sigma$ is an element of the centre of $\mathcal{M}(M, P)$ and thus an element of $\mathcal{Z}_{G}(H)$. Finally, from the equality $\mathcal{N}_{G}(H)=\operatorname{Stab}(N) \times\langle\sigma\rangle$ of Theorem 7.1 follows the equality $\mathcal{Z}_{G}(H)=\mathcal{M}(N, N \cap P) \times\langle\sigma\rangle$.

\section{References}

[1] J. Birman, "Braids, links, and mapping class groups", Annals of Mathematics Studies 82, Princeton University Press and University of Tokyo Press, Princeton, 1975.

[2] J. Birman, Mapping class groups of surfaces, Braids, AMS-IMS-SIAM Jt. Summer Res. Conf., Santa Cruz/Calif. 1986, Contemp. Math. 78, 1988, pp. $13-43$.

[3] J. Birman, A. Lubotzky, J. McCarthy, Abelian and solvable subgroups of the mapping class group, Duke Math. J. 50 (1983), 1107-1120. 
[4] W. Chow, On the algebraical braid group, Ann. Math. 49 (1948), 654-658.

[5] D.B.A. Epstein, Curves on 2-manifolds and isotopies, Acta Math. 115 (1966), 83-107.

[6] D.B.A. Epstein, J. W. Cannon, D. Holt, S. Levy, M. Paterson, W. Thurston, "Word processing in groups," Jones and Bartlett, Boston, 1992.

[7] A. Fathi, F. Laudenbach, and V. Poénaru, "Travaux de Thurston sur les surfaces, Séminaire Orsay", Astérisque 66-67, 1979.

[8] R. Fenn, D. Rolfsen, and J. Zhu, Centralisers in the braid group and singular braid monoid, Enseign. Math. 42 (1996), 75-96.

[9] S. Gervais, A finite presentation of the mapping class group of an oriented surface, preprint, 1998.

[10] E. Grossman, On the residual finiteness of certain mapping class groups, J. London Math. Soc. 9 (1974), 160-164.

[11] A. Hatcher, W. Thurston, A presentation for the mapping class group of a closed orientable surface, Topology 19 (1980), 221-237.

[12] A. Hurwitz, Über algebraische Gebilde mit eindeutige Transformationen in sich, Math. Ann. 41 (1893), 403-442.

[13] A. Ishida, The structure of subgroups of mapping class groups generated by two Dehn twists, Proc. Japan Acad. Ser. A 72 (1996), 240-241.

[14] N.V. Ivanov, Complexes of curves and the Teichmüller modular group, Russ. Math. Surv. 42 (1987), 55-107.

[15] N.V. Ivanov, "Subgroups of Teichmüller modular groups", Translation of Mathematical Monographs 115, Amer. Math. Soc., Providence, 1992.

[16] N.V. Ivanov, Automorphisms of Teichmüller modular groups, Topology and geometry, Rohlin Semin. 1984-1986, Lect. Notes Math. 1346, Springer-Verlag, Berlin-Heidelberg-New York, 1988, pp. 199-270.

[17] N.V. Ivanov, J.D. McCarthy, On injective homomorphisms between Teichmüller modular groups I, Invent. Math. 135 (1999), 425-486.

[18] D. Johnson A survey of the Torelli group, Low-dimensional topology (San Francisco, Calif., 1981), Contemp. Math., 20, Amer. Math. Soc., Providence, R.I., 1983, 165-179.

[19] D. Johnson, The structure of the Torelli group. I. A finite set of generators for $\mathcal{I}$, Ann. of Math. (2) 118 (1983), no. 3, 423-442. 
[20] D. Johnson, The structure of the Torelli group. II. A characterization of the group generated by twists on bounding curves, Topology 24 (1985), no. 2, $113-126$.

[21] D. Johnson, The structure of the Torelli group. III. The abelianization of $\mathcal{I}$, Topology 24 (1985), no. 2, 127-144.

[22] S. Kerckhoff, The Nielsen realization problem, Ann. Math. 117 (1983), 235265.

[23] W.B.R. Lickorish, A finite set of generators for the homeotopy group of a 2-manifold, Proc. Camb. Philos. Soc. 60 (1964), 769-778.

[24] R.C. Lyndon, P.E. Schupp, "Combinatorial group theory", Springer-Verlag, Berlin-Heidelberg-New York, 1977.

[25] E. Looijenga, Affine Artin groups and the fundamental groups of some moduli spaces, preprint, 1998.

[26] M. Matsumoto, A presentation of mapping class groups in terms of Artin groups and geometric monodormy of singularities, preprint, 1998.

[27] J.D. McCarthy, A Tits alternative for subgroups of surface mapping class groups, Trans. Amer. Math. Soc. 291 (1985), 583-612.

[28] J.D. McCarthy, Automorphisms of surface mapping class groups. A recent theorem of N. Ivanov, Invent. Math. 84 (1986), 49-71.

[29] J. McCool, Some finitely presented subgroups of the automorphism group of a free group, J. Algebra 35 (1975), 205-213.

[30] G. Mislin, Mapping class groups, characteristic classes, and Bernouilli numbers, CRM Proc. Lect. Notes 6 (1994), 103-131.

[31] S. Morita, Mapping class groups of surfaces and three-dimensional manifolds, Proc. Int. Congr. Math., Kyoto/Japan 1990, Vol. I, 1991, pp. 665-674.

[32] L. Mosher, Mapping class groups are automatic, Ann. Math. 142 (1995), 303384.

[33] V.P. Platonov, Frattini subgroups of linear groups and finitary approximability, Dokl. Akad. Nauk SSSR 171 (1966), 798-801; English transl., Soviet Math. Dokl. 7 (1966), 1557-1560.

[34] J. Powell, Two theorems on the mapping class group of a surface, Proc. Amer. Math. Soc. 68 (1978), 347-350.

[35] D. Rolfsen, Braid subgroup normalisers, commensurators and induced representations, Invent. Math. 130 (1997), 575-587. 
[36] B. Wajnryb, A simple presentation for the mapping class group of an orientable surface, Isr. J. Math. 45 (1983), 157-174.

[37] B. Wajnryb, Mapping class group of a surface is generated by two elements, Topology 35 (1996), 377-383.

[38] H. Zieschang, Finite groups of mapping classes of surfaces, Lect. Notes Math. 875, Springer-Verlag, Berlin-Heidelberg-New York, 1981.

\section{Luis PARIS}

Laboratoire de Topologie

Université de Bourgogne

UMR $5584 \mathrm{du}$ CNRS

BP 47870

21078 Dijon Cedex

FRANCE

lparis@u-bourgogne.fr

\section{Dale ROLFSEN}

Department of Mathematics

University of British Columbia

Vancouver

British Columbia V6T 1Z2

CANADA

rolfsen@math.ubc.ca 